\title{
Fluids and fluid mixtures containing square-well diatomics: Equations of state and canonical molecular dynamics simulation
}

\author{
Harpreet S. Gulati and Carol K. Hall \\ Department of Chemical Engineering, North Carolina State University, Raleigh, \\ North Carolina 27695-7905
}

(Received 4 April 1997; accepted 6 June 1997)

\begin{abstract}
We present new perturbation theory equations of state for square-well dimer fluids, square-well dimer mixtures, square-well dimer/monomer mixtures and square-well heteronuclear dumbbell fluids. Our first- and second-order perturbation terms are based on Barker and Henderson's local compressibility approximation and Chang and Sandler's perturbation theory, respectively. The perturbation approach requires knowledge of the radial distribution functions of the reference hard-dimer fluid and hard dimer/monomer mixture, which are obtained from molecular dynamics simulation. For mixtures we use one fluid mixing rules to approximate the average mixture structure and perturbation parameters. The predictions of the perturbation theory are compared to the compressibility factors obtained from discontinuous canonical molecular dynamics simulation, an adaptation of Anderson's canonical ensemble molecular dynamics method to the case in which the potential is discontinuous. (C) 1997 American Institute of Physics. [S0021-9606(97)51634-5]
\end{abstract}

\section{INTRODUCTION}

In this laboratory we have been attempting to develop equations of state for copolymers (heteronuclear chain fluids) and for polymer blends (homonuclear chain mixtures). As a first step towards these goals we recently developed new generalized Flory-Dimer theories for hard heteronuclear chain fluids ${ }^{1}$ and for hard-chain mixtures. ${ }^{2}$ However, in order to develop a theory that will be suitable for practical applications it is necessary to incorporate attractive interactions into these models. The simplest way to do this is to extend our generalized Flory-Dimer treatment to heteronuclear chain fluids and to homonuclear chain mixtures whose segments interact via square-well attractions. This requires the development of analytical equations of state for square-well monomer mixtures, square-well dimer mixtures, and squarewell heteronuclear dumbbell fluids.

Owing to their theoretical importance, fluids composed of particles interacting via a site-site square-well potential have been the subject of intense interest. The pure squarewell monatomic (monomer) fluid has been studied extensively by both computer simulation ${ }^{3-6}$ and theoretical methods. $^{7-9}$ More recent efforts, however, have been devoted to the study of square-well monomer mixtures. Several accurate equations of state based on local composition theory have been proposed for binary ${ }^{10-14}$ and ternary ${ }^{15}$ square-well mixtures. Bokis and Donohue ${ }^{16}$ have recently proposed an accurate perturbation theory for binary mixtures of equalsized molecules. Extensive Monte Carlo simulation studies of binary ${ }^{10,12}$ and ternary ${ }^{15}$ square-well mixtures have also been conducted in order to evaluate these theories. Squarewell dimer fluids have also been investigated ${ }^{17-19}$ due to their utility as a building block in theories of square-well chain fluids. ${ }^{20,21}$ Yethiraj and Hall ${ }^{17}$ used the reference interaction site model and perturbation theory to develop two new equations of state for square-well dimer fluids. Thomas and Donahue $^{19}$ used the local composition model and perturba- tion theory to obtain the thermodynamic properties of overlapping square-well dimers of variable bond length.

In this paper we obtain equations of state for four cases: 1) pure fluids containing homonuclear dimers, 2) mixtures of square-well dimers, 3) mixtures of square-well dimers and square-well monomers, and 4) pure fluids containing squarewell heteronuclear dumbbells. The new equations of state are based on the perturbation theory approach. ${ }^{7,22}$ In order to test the accuracy of these new equations of state, we also perform extensive discontinuous canonical molecular dynamics (DCMD) simulations and compare the resulting compressibility factors to our theoretical predictions.

In the perturbation theory approach, the free energy of a square-well (SW) fluid is expanded in a series in inverse temperature about the free energy of a reference fluid (usually the corresponding hard-sphere fluid) whose structure and thermodynamic properties are known. For the SW dimer fluid, SW dimer mixture, SW dimer/monomer mixture, and SW heteronuclear dumbbell fluid, the reference fluids are the corresponding hard-dimer fluid, hard-dimer mixture, hard dimer/monomer mixture, and hard-heteronuclear dumbbell fluid, respectively. In this paper the thermodynamic properties of all of the reference hard fluids and mixtures are obtained from the scaled particle theory equation of state. ${ }^{23,24}$ The first- and second-order perturbation terms are calculated using Barker and Henderson's ${ }^{7}$ local compressibility approximation and Chang and Sandler's ${ }^{22}$ perturbation approach, respectively. The first- and second-order perturbation terms require expressions for the volume integrals of the reference fluid's radial distribution functions (RDF). For hardhomonuclear dimer fluids and for hard dimer/monomer mixtures where all of the segments are of the same size, this structural information is deduced from the radial distribution functions calculated from discontinuous molecular dynamics (DMD) simulation. ${ }^{1}$ For hard dimer/monomer mixtures where the dimer and monomer segments are of different 
sizes, we approximate these integrals with the corresponding integrals for the case where the dimer and monomer segments are of the same size. Similarly, for hard-dimer mixtures and for hard heteronuclear dumbbell fluids where the segments of the two components are of different sizes, we approximate these integrals with the corresponding integrals for hard-homonuclear dimer fluids at the same density as the mixture. We also use conformal-fluid mixing rules, proposed by Henderson ${ }^{25}$ and Hino and Prausnitz, ${ }^{26}$ to calculate the average mixture parameters that are used in the perturbation theory.

The rest of the paper is organized as follows: Section II briefly describes the molecular model and the computer simulation method used to study the four cases described above. Section III reviews the application of perturbation theory to square-well monomer fluids and then describes its extension to square-well dimer fluids, square-well dimer mixtures, square-well dimer/monomer mixtures, and squarewell heteronuclear dumbbell fluids. Section IV discusses the accuracy of the new perturbation-theory-based equations of state in predicting the compressibility factors of square-well dimer fluids, square-well dimer mixtures, square-well dimer/ monomer mixtures, and square-well heteronuclear dumbbell fluids by comparing theoretical predictions to simulation results.

\section{MOLECULAR MODEL AND SIMULATION METHOD}

In this section we describe the molecular model used and the simulation method employed to study square-well dimer fluids, square-well dimer mixtures, square-well dimer/ monomer mixtures and square-well heteronuclear dumbbell fluids. The site-site potential energy between two segments, $i$ and $j$, interacting via a square-well potential, is given by

$$
\begin{aligned}
u_{i j}(r) & =\infty, \quad 0<r<\sigma_{i j} \\
& =-\epsilon_{i j}, \quad \sigma_{i j}<r \lambda_{i j} \sigma_{i j} \\
& =0, \quad r>\lambda_{i j} \sigma_{i j},
\end{aligned}
$$

where $\sigma_{i j}=\left(\sigma_{i}+\sigma_{j}\right) / 2$ is the center-to-center distance between segments $i$ and $j, \lambda_{i j}$ is the square-well width, and $\epsilon_{i j}$ is the cross interaction energy between segments $i$ and $j$, taken to be

$$
\epsilon_{i j}=\sqrt{\epsilon_{i} \epsilon_{j}} .
$$

Although the square-well width for realistic fluids has been determined to be approximately equal to $1.4,{ }^{27}$ most theoretical work has focussed on $\lambda=1.5 .^{3,10,28}$ In order to be consistent with the bulk of the theoretical work available for square-well fluids, we assume a square-well width of $\lambda_{i j}$ $=\lambda=1.5$ for all cases.

Figure 1 illustrates the four types of systems investigated here: SW dimer fluids, SW dimer mixtures, SW dimer/ monomer mixtures, and SW heteronuclear dumbbell fluids. The square-well dimer fluid contains tangent square-well diatomic molecules with segment diameter $\sigma$ and well width $\epsilon$. The square-well dimer mixture contains two species (1 and 2) of tangent square-well diatomic molecules with diameter $\sigma_{1}$ and well depth $\epsilon_{1}$ (species 1), and diameter $\sigma_{2}$ and well depth $\epsilon_{2}$ (species 2), respectively. The square-well (a)

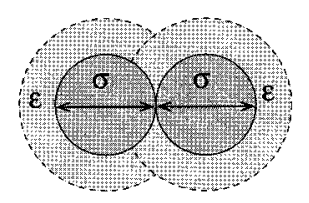

(b)

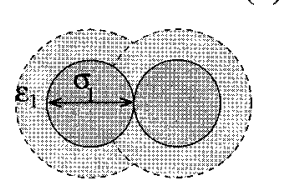

(c)
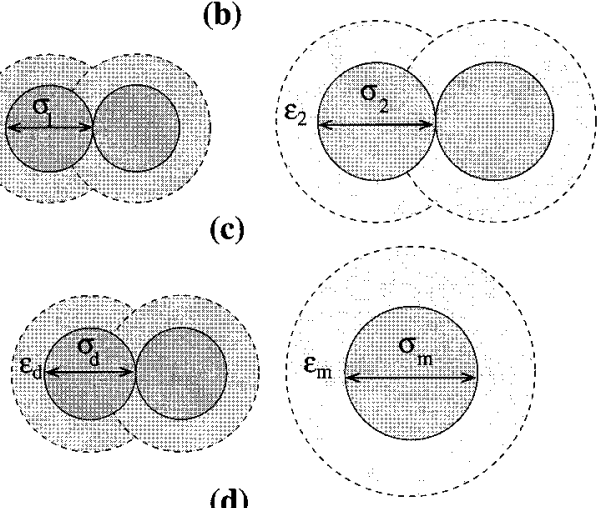

(d)

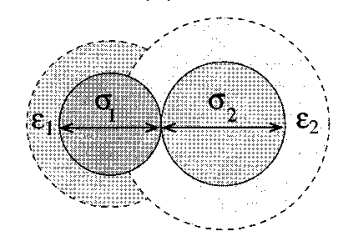

FIG. 1. Four types of square-well systems studied: (a) square-well dimer fluids, (b) square-well dimer mixtures, (c) square-well dimer/monomer mixtures, and (d) square-well heteronuclear dumbbell fluids.

dimer/monomer mixture contains square-well homonuclear diatomics of diameter $\sigma_{d}$ and well depth $\epsilon_{d}$, and square-well monomers of diameter $\sigma_{m}$ and well depth $\epsilon_{m}$. The squarewell dumbbell fluid contains heteronuclear tangent squarewell diatomic molecules each of which contains a segment of species 1 (diameter $\sigma_{1}$ and well depth $\epsilon_{1}$ ) and a segment of species 2 (diameter $\sigma_{2}$ and well depth $\epsilon_{2}$ ).

The simulations technique used throughout our work is discontinuous molecular dynamics (DMD), ${ }^{29}$ a variant on the standard molecular dynamics technique that is applicable to systems of molecules interacting via discontinuous potentials, e.g. hard-spheres and square-well spheres. DMD on diatomic molecules containing tangent hard spheres (the hard dimer model) or tangent square-well spheres (the square-well dimer model) is implemented using the bead-string algorithm introduced by Rapaport $^{30,31}$ and later modified by Bellemans. ${ }^{32}$ In the Rapaport algorithm, dimer connectivity is maintained by linking adjacent spheres on a dimer with short invisible strings. The length of this string is allowed to vary freely over the range between $\sigma$ and $(1+\delta) \sigma$, where $\delta \ll 1$. This effectively decouples the motion of bonded spheres on the dimer, allowing them to move independently along linear trajectories between core collisions and bondstretch collisions. Following Bellemans et al. ${ }^{32}$ we permit slight interpenetration of neighboring (bonded) spheres so that their separation distance fluctuates between $(1-\delta / 2) \sigma$ and $(1+\delta / 2) \sigma$, resulting in an average bond length of $\sigma$. 
Square-well interactions are accommodated in the Rapaport algorithm by introducing "well-capture" and "wellbounce" collisions which occur whenever a sphere enters or leaves the square well of any other sphere.

Discontinuous molecular dynamics is conventionally conducted in a microcanonical ensemble (constant $N, V$, $E$ ) where the Newtonian equations of motion of a set of $N$ particles in volume $V$ are solved numerically. The total energy $E$ of the system is conserved and the temperature $T$ fluctuates about a mean value as the system evolves along its trajectory. Details of the microcanonical discontinuous molecular dynamics (DMD) have been presented by Alder and Wainwright. ${ }^{29}$ The DMD algorithm exploits the fact that the trajectory of the particles changes only when the particleparticle separation coincides with a discontinuity in their pair potential, e.g. when a core collision, a bond collision, or a square-well collision occurs. This implies that the phase trajectory of the system develops on an event-by-event basis. Thus, the DMD algorithm proceeds by calculating the order in which collisions will occur, advancing the system to the imminent collision, and calculating the post-collision velocities of the participating particles.

We have adapted DMD to the canonical (constant $N$, $V, T$ ) ensemble using a discontinuous potential version of Anderson's method. ${ }^{33-35}$ The essential feature of Anderson's method is the introduction of stochastic energy fluctuations into an NVE ensemble such that the trajectory average of any property is equal to its NVT ensemble average. We assume that the system is immersed in an imaginary constant temperature heat bath containing imaginary "ghost" particles. The velocities of these ghost particles are assigned according to a Maxwell-Boltzmann distribution about the required temperature. The energy fluctuations are introduced via the interaction of these ghost particles with the system particles. The ghost particles regulate the system temperature by colliding with system particles and exchanging their velocities. Between two successive collisions with ghost particles, the ensemble trajectory evolves in accordance with microcanonical ensemble dynamics. Effectively, we periodically reassign the velocities of the system particles according to a MaxwellBoltzmann distribution about the required temperature. Thus, Anderson's method is implemented for our DMD method by invoking a new type of collision, henceforth called the "ghost" collision, in addition to the core, bond, and squarewell collisions.

To perform the discontinuous canonical molecular dynamics (DCMD) simulation, we have to first choose two parameters: $T$, the desired simulation temperature and $\nu$, the mean rate at which a ghost collision occurs. We then determine the ghost collision times for each particle using these two parameters. Since, the heat bath particles are not real, we cannot calculate ghost collision times using equations of motion. Instead, we assign the ghost collision times using probability arguments. The time intervals between ghost collisions are distributed according to the exponential distribution $^{33}$

$$
P(t)=\nu e^{-\nu t},
$$

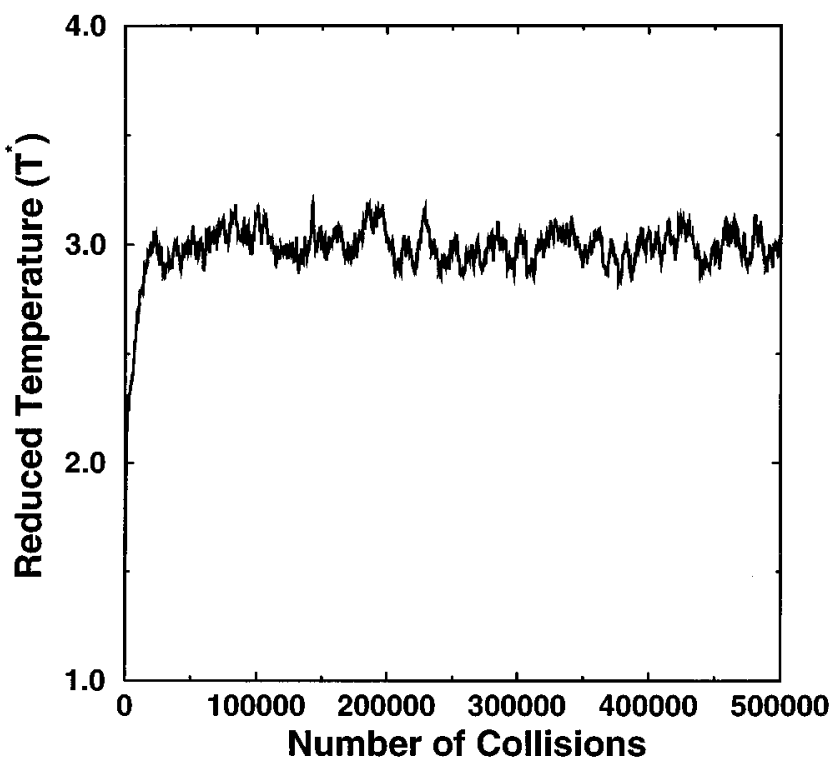

FIG. 2. The fluctuations in temperature observed in the first 50000 collisions during DCMD simulation of a square-well dimer fluid at $\eta=0.3$. The system contains $N_{s}=512$ segments and is relaxed from an initial temperature $T^{*}=1$ to the final simulation temperature $T^{*}=3$.

where $P(t) \Delta t$ is the probability that any particle experiences a ghost collision in the interval $t$ and $t+\Delta t$. Thus, the ghost collision time for each particle is determined using random numbers generated according to an exponential distribution. ${ }^{36}$ The mean-squared fluctuation of the total energy of the system, and hence the equilibrium properties, are independent of the value of $\nu \cdot{ }^{33}$ However, the time dependence of the fluctuation of the energy is very sensitive to the value of $\nu$. Therefore, $\nu$ should be chosen such that the time for the decay of energy fluctuations mimics the time to relax the energy of a small volume of real liquid surrounded by a much larger volume, i.e. the ghost collision rate should be similar to the mean collision rate of the system particles. The mean collision rate of the ghost particles can be determined using the kinetic theory of gases ${ }^{34,37}$

$$
\nu=\rho_{g} \sigma^{2} \sqrt{16 \pi k T / m},
$$

where $\rho_{g}$ is the number density of ghost particles, $k$ is the Boltzmann constant, and $m$ is the mass of a ghost particle which is assumed to be the same as the mass of a system particle. Reduced ghost densities, $\rho_{g}^{*}=\rho_{g} \sigma^{3}$, of about 0.5 yields approximately $10-15$ ghost collisions for every 100 system collisions (core-collisions, bond-collisions and square-well events) and is sufficient to generate a constanttemperature trajectory. Figure 2 shows the DCMD temperature fluctuation behavior for a square-well dimer fluid at $T^{*}=3, \quad \rho_{g}^{*}=0.5$ and $\eta=0.3$, where $T^{*}=k T / \epsilon$ and $\eta$ $=\pi N_{s} \sigma^{3} / 6$. The ensemble consists of $N_{s}=512$ segments and has an initial temperature $T^{*}=1$.

Once the temperature $(T)$ and the mean-collision rate $(\nu)$ for the system are determined, the ghost collision time for each particle is assigned as explained above. The times for core-collision, bond-collision, and square-well collision 
for each pair of particles in the system are calculated by solving the equations of motion. The DCMD simulation algorithm develops on a collision-by-collision basis by locating the next event (core collision, bond collision, square-well collision, or ghost collision) in the system, advancing the system to that point in time, computing the collision dynamics, and repeating the process. At each simulation step or event, the velocities of the particles involved in that event change in both magnitude and direction. The core-collisions, bond-collisions, and square-well collisions involve two system particles whereas ghost collisions involve only one system particle. As a result of the event, new core collision times, bond collision times, and square-well collision times are computed, and new ghost collision times assigned for the particles involved in the event.

The DCMD simulations are performed at volume fractions $\eta$ ranging from 0.09 to 0.45 where $\eta=\pi \Sigma N_{i} \sigma_{i}^{3} /(6 V)$ with $N_{i}$ equal to the number of segments of species $i$ and $V$ equal to the volume of the primary simulation cell. A system size of $N_{s}=512$ segments has been used for all cases. The compressibility factors are obtained for a wide range of cases with diameter ratios of the two components ranging from 0.5 to 2 and well depth ratios, $\epsilon_{2} / \epsilon_{1}$, ranging from 0.5 to 1.5 . All systems are studied at reduced temperatures ranging from 1.5 to 3 . We have confined our simulations to these relatively high temperatures because the system may phase separate at lower temperatures leading to ambiguous simulation results. Although, we only present results for binary mixtures and heteronuclear diatomic fluids composed of two types of segments, the DCMD method is also applicable to multicomponent mixtures and heteronuclear fluids composed of more than two types of segments.

The compressibility factor is calculated using an equation derived from the virial theorem of Clausius ${ }^{38}$

$$
Z=\frac{N_{s}}{N_{t}}-\frac{1}{3 N_{t} k T t_{e}} \sum_{\text {colls }} m_{i} \mathbf{r}_{\mathbf{i j}} \cdot \Delta \mathbf{v}_{\mathbf{i}}
$$

where $N_{s}$ is the total number of segments in the system and $N_{t}$ is the total number of molecules in the system. Here $\mathbf{r}_{\mathbf{i j}}=\mathbf{r}_{\mathbf{i}}-\mathbf{r}_{\mathbf{j}}$, where $\mathbf{r}_{\mathbf{i}}$ and $\mathbf{r}_{\mathbf{j}}$ are the position vectors of the colliding particles $i$ and $j$. The quantity $m_{i} \Delta \mathbf{v}_{\mathbf{i}}$ is the collisional impulse experienced by particle $i$ and is a measure of the force exerted by particle $j$ on particle $i$. The collisional virial is summed over all intermolecular and intramolecular core collisions, bond stretches and square-well collisions occurring during the elapsed simulation time $t_{e}$. Since ghost collisions involve only one system particle, they do not contribute to the collisional virial.

The efficiency of the simulation is measured in terms of the number of collisions generated per unit CPU time. Various DMD efficiency-enhancing schemes have been used to optimize the performance of our code. These include neighbor lists, ${ }^{39}$ single-event time list, ${ }^{36,40}$ delayed position updating, ${ }^{41}$ link lists, ${ }^{42}$ and binary tree searches. Our simulation code ran at approximately 5 million collisions per CPU hour on DEC Alpha 3000/300LX workstations for a system with $N_{s}=512$ segments. The duration of the simulation runs range from 10 million collisions at high temperatures to 30 million collisions at low temperatures. The error bars on the compressibility factors calculated in these simulations represent \pm 2 standard deviations as determined from three or more independent runs. In the cases where the error bars are omitted, the error is smaller than $0.1 \%$.

\section{PERTURBATION THEORY}

The concept that underlies the perturbation approach is that the free energy of a system can be expressed as an expansion in the inverse temperature around the free energy of a reference system whose structure and thermodynamic properties are known. The second order perturbation theory expansion of the Helmholtz free energy is of the form

$$
\frac{A^{\mathrm{ex}}}{N k T}=\frac{A_{0}^{\mathrm{ex}}}{N k T}+\left(\frac{1}{k T}\right) \frac{A_{1}}{N k T}+\left(\frac{1}{k T}\right)^{2} \frac{A_{2}}{N k T},
$$

where $A_{0}^{\text {ex }}$ is the excess Helmholtz free energy of the reference system, $A_{1}$ and $A_{2}$ are the first- and second-order perturbation terms, respectively, and $N$ is the number of molecules in the system. The first- and second-order perturbation terms are derived from knowledge of the thermodynamic properties (equation of state) and the structure (radial distribution function) of the reference fluid. In this section we present new equations of state based on perturbation theory for square-well dimer fluids, square-well dimer mixtures, square-well dimer/monomer mixtures and square-well heteronuclear dumbbell fluids. We begin with a brief review of the more successful approaches to calculating the first- and second-order perturbation terms for homonuclear squarewell monomer (standard square-well fluid) and dimer fluids, and then present our perturbation-theory equations of state for square-well dimer fluids, square-well dimer mixtures, square-well dimer/monomer mixtures, and square-well heteronuclear dumbbell fluids.

\section{A. Square-well monomer fluid}

For the square-well fluid, the natural choice for the reference system is the hard sphere fluid. Barker and Henderson (BH) (Refs. 7, 25) used second order perturbation theory to express the excess Helmholtz free energy of a square-well monomer fluid in terms of the excess Helmholtz free energy and radial distribution function (RDF) of a hard-sphere fluid.

According to Barker and Henderson, the first-order perturbation contribution is given $\mathrm{by}^{7}$

$$
\frac{A_{1}}{N k T}=\frac{\rho}{2} \int_{0}^{\infty} u_{1}(r) g_{0}(r) 4 \pi r^{2} d r,
$$

where $g_{0}(r)$ is the reference hard-monomer site-site RDF, $\rho=N / V$ is the density, and $u_{1}(r)=u(r)-u_{0}(r)$ is the perturbation in the site-site interaction potential. For the squarewell monomer fluid, the first-order perturbation term reduces to

$$
\frac{A_{1}^{\mathrm{mon}}}{N k T}=-2 \pi \rho \sigma^{3} \epsilon I_{0}^{\mathrm{mon}},
$$


where

$$
I_{0}^{\mathrm{mon}}=\int_{1}^{\lambda} g_{0}(x) x^{2} \mathrm{~d} x
$$

with $x=r / \sigma$. The second-order perturbation term is calculated using the local compressibility approximation of Barker and Henderson ${ }^{7}$

$$
\frac{A_{2}}{N k T}=-\frac{\rho}{4} \frac{\partial}{\partial p_{0}}\left[\rho \int_{0}^{\infty} u_{1}^{2} g_{0}(r) 4 \pi r^{2} d r\right],
$$

where $p_{0}$ is the pressure of the reference hard-sphere fluid. For the square-well monomer fluid the above expression reduces to

$$
\frac{A_{2}^{\mathrm{mon}}}{N k T}=-\pi \rho \sigma^{3} \epsilon^{2} \frac{\partial \rho}{\partial p_{0}^{\mathrm{mon}}} \times \frac{\partial\left(\eta I_{0}^{\mathrm{mon}}\right)}{\partial \eta} .
$$

The second-order perturbation term may also be calculated using the superposition approximation of Smith et al., ${ }^{43}$ however the local compressibility approximation term is preferred since it results in analytical expressions for the second order term. ${ }^{22}$ Barker and Henderson ${ }^{7}$ obtained the hardsphere excess Helmholtz free energy and $\partial \rho / \partial p_{0}^{\text {mon }}$ from the Percus-Yevick (PY) “compressibility" equation of state. The hard-sphere radial distribution function, $g_{0}(r)$, was calculated by solving the PY equation.

Chang and Sandler ${ }^{44}$ recently derived a new accurate equation of state for square-well monomer fluids based on perturbation theory. They obtained a completely analytical solution for the radial distribution function of hard spheres and used this explicit expression to evaluate the integrand $I_{0}$ in terms of the packing fraction $\eta$ and well width $\lambda$. Chang and Sandler used the Carnahan-Starling ${ }^{45}$ equation of state for the hard-sphere excess Helmholtz free energy and the PY compressibility equation to calculate $\partial \rho / \partial p_{0}$ thereby obtaining an expression for the excess Helmholtz free energy of a square-well monomer fluid. Their expression for the compressibility factor, which is obtained by differentiating the Helmholtz free energy with respect to $\eta$, is given by

$$
Z_{\mathrm{sw}}^{\mathrm{mon}}=\eta \frac{\partial}{\partial \eta}\left(\frac{A^{\mathrm{ex}}}{N k T}\right)=Z_{0}^{\mathrm{mon}}+\left(\frac{1}{k T}\right) Z_{1}^{\mathrm{mon}}+\left(\frac{1}{k T}\right)^{2} Z_{2}^{\mathrm{mon}},
$$

where

$$
\begin{aligned}
& Z_{0}^{\mathrm{mon}}=\frac{p_{0}^{\mathrm{mon}}}{\rho k T}=\frac{1+\eta+\eta^{2}-\eta^{3}}{(1-\eta)^{3}}, \\
& Z_{1}^{\mathrm{mon}}=-12 \eta \epsilon\left(I_{0}^{\mathrm{mon}}+\eta \frac{\partial I_{0}^{\mathrm{mon}}}{\partial \eta}\right),
\end{aligned}
$$

$$
\begin{aligned}
Z_{2}^{\mathrm{mon}}= & -\frac{6 \epsilon^{2} \eta(1-\eta)^{3}\left(1-5 \eta-20 \eta^{2}-12 \eta^{3}\right)}{\left(1+4 \eta+4 \eta^{2}\right)^{2}} \\
& \times\left(I_{0}^{\mathrm{mon}}+\eta \frac{\partial I_{0}^{\mathrm{mon}}}{\partial \eta}\right)-\frac{6 \epsilon^{2} \eta^{2}(1-\eta)^{4}}{\left(1+4 \eta+4 \eta^{2}\right)} \\
& \times\left(2 \frac{\partial I_{0}^{\mathrm{mon}}}{\partial \eta}+\eta \frac{\partial^{2} I_{0}^{\mathrm{mon}}}{\partial \eta^{2}}\right) .
\end{aligned}
$$

Although, the second order perturbation term is generally small in magnitude, it improves the accuracy of the perturbation theory at very low and very high densities. Chang and Sandler calculated the compressibility factor, critical properties, and vapor-liquid equilibrium of square-well monomers for well widths ranging from 1 to 2 and found very good agreement between their predictions and Gibbs ensemble Monte Carlo results. ${ }^{46}$

\section{B. Square-well dimer fluid}

Yethiraj and Hall ${ }^{17}$ used the BH perturbation approach to obtain an expression for the compressibility factor of a square-well dimer fluid. The reference fluid was taken to be the hard-dimer fluid. Yethiraj and Hall calculated the firstand second-order perturbation terms by performing Monte Carlo simulations of the structure of a hard-dimer reference fluid. More specifically, they calculated $N_{\lambda}$, the number of pairs of sites within a distance $\lambda \sigma$ of each other. The two perturbation terms are then

$$
\frac{A_{1}}{N k T}=-4 \epsilon \frac{\rho}{2} \int_{\sigma}^{\lambda \sigma} 4 \pi g_{0}(r) r^{2} d r=-\frac{\left\langle N_{\lambda}\right\rangle_{0}}{N k T}
$$

and

$$
\frac{A_{2}}{N k T}=-\frac{\left\langle N_{\lambda}^{2}\right\rangle_{0}-\left\langle N_{\lambda}\right\rangle_{0}^{2}}{N k T},
$$

where \langle\rangle$_{0}$ denotes an ensemble average over all configurations of the reference system, and $g_{0}$ is the site-site pair correlation function of the reference hard-dimer fluid. The factor " $4 \epsilon$ " in Eq. 16 is due to the fact that for every pair of dimer molecules, there are 4 attractive site-site interactions. Yethiraj and Hall fitted the first-order Helmholtz free energy perturbation term to a function of $\eta$ and, by differentiating it with respect to $\eta$ were able to obtain the first-order perturbation term for the compressibility factor

$$
Z_{1}^{\operatorname{dim}}=12.003 \eta \frac{1+1.566 \eta-15.129 \eta^{2}+19.147 \eta^{3}-4.787 \eta^{3}}{(1-\eta)^{4}} .
$$

The second-order Helmholtz free energy perturbation term was fitted using an expression similar to the "energydensity" expression proposed by Lee et al. ${ }^{10}$ and differentiated with respect to $\eta$ to obtain the second-order perturbation term for the compressibility factor

$$
Z_{2}^{\mathrm{dim}}=492.36296 \eta^{2} \frac{(2-12.31907 \eta)}{(1+8.26765 \eta)^{4}}
$$


Thomas and Donohue ${ }^{19}$ later developed an approximate method for calculating the second-order term based on knowledge of the first-order term, hence avoiding having to fit simulation data to obtain the second-order term. Both theories are found to accurately predict the compressibility factor of square-well dimer fluids over a wide range of densities and temperatures.

We extend Chang and Sandler's ${ }^{22}$ perturbation theory approach to square-well dimer fluids. Following Eqs. 7 and 11 , the first- and second-order perturbation terms for the Helmholtz free energy of square-well dimers are given as

$$
\frac{A_{1}^{\mathrm{dim}}}{N k T}=-2 \pi \rho \sigma^{3} 4 \epsilon I_{0}^{\mathrm{dim}}
$$

and

$$
\frac{A_{2}^{\operatorname{dim}}}{N k T}=-\pi \rho \sigma^{3} 4 \epsilon^{2} \frac{\partial \rho}{\partial p_{0}^{\operatorname{dim}}} \times \frac{\partial\left(\eta I_{0}^{\mathrm{dim}}\right)}{\partial \eta},
$$

where

$$
I_{0}^{\operatorname{dim}}=\int_{1}^{\lambda} g_{0}^{\operatorname{dim}}(x) x^{2} \mathrm{~d} x
$$

is the integral of the radial distribution function of a harddimer fluid, $g_{0}^{\text {dim }}$ is the hard-dimer site-site radial distribution function and $x=r / \sigma$. The hard-dimer radial distribution function, $g_{0}^{\mathrm{dim}}$, and it's integral, $I_{0}^{\mathrm{dim}}$, are functions of density (or packing fraction) only. As explained earlier, the factor 4 in Eqs. 20 and 21 is due to the fact that there are 4 attractive site-site interactions for each pair of dimer molecules. For a more generalized notation this factor is replaced by $n^{2}$, where $n$ is the number of segments per molecule ( $n$ $=2$ for a dimer). The reference hard-dimer Helmholtz free energy $\left(A_{0}^{\mathrm{dim}}\right)$ and the pressure $\left(p_{0}^{\mathrm{dim}}\right)$ are calculated from the scaled particle theory equation of state. Although, the Tildesley and Street ${ }^{47}$ hard-dimer equation of state can be used to calculate the $A_{0}^{\mathrm{dim}}$ and $p_{0}^{\mathrm{dim}}$, we prefer to use the scaled particle theory equation of state because the perturbation expressions derived using scaled particle theory can also be used for hard dimer mixtures, hard dimer/monomer mixtures and hard heteronuclear dumbbell fluids.

The perturbation terms for the compressibility factor of a square-well dimer fluid are obtained by differentiating the corresponding Helmholtz free energy term with respect to $\eta$,

$$
Z_{\mathrm{sw}}^{\mathrm{dim}}=\eta \frac{\partial}{\partial \eta}\left(\frac{A_{\mathrm{dim}}^{\mathrm{ex}}}{N k T}\right)=Z_{0}^{\mathrm{dim}}+\left(\frac{1}{k T}\right) Z_{1}^{\mathrm{dim}}+\left(\frac{1}{k T}\right)^{2} Z_{2}^{\mathrm{dim}},
$$

where $Z_{0}^{\mathrm{dim}}$ is the reference hard-dimer fluid compressibility factor and $Z_{1}^{\mathrm{dim}}$ and $Z_{2}^{\mathrm{dim}}$ are the first- and second-order perturbation terms for the compressibility factor of a squarewell dimer fluid. The reference hard-dimer fluid compressibility is obtained from the scaled particle theory expression,

$$
Z_{0}^{\mathrm{dim}}=\frac{p_{0}^{\mathrm{dim}}}{\rho k T}=\frac{1}{1-\eta}+\frac{3 \alpha \eta}{(1-\eta)^{2}}+\frac{3 \alpha^{2} \eta^{2}-\alpha^{2} \eta^{3}}{(1-\eta)^{3}},
$$

TABLE I. Geometric parameters for convex bodies.

\begin{tabular}{lccc}
\hline \hline Convex body & Volume $(b)$ & Surface area $(s)$ & $\begin{array}{c}\text { Radius of } \\
\text { curvature }(r)\end{array}$ \\
\hline Monomer & $\frac{\pi \sigma^{3}}{6}$ & $\pi \sigma^{2}$ & $\frac{\sigma}{2}$ \\
Homonuclear & $\frac{\pi \sigma^{3}}{3}$ & $2 \pi \sigma^{2}$ & $\frac{3 \sigma}{4}$ \\
Dimer & $\frac{\pi\left(\sigma_{1}^{3}+\sigma_{2}^{3}\right)}{6}$ & $\pi\left(\sigma_{1}^{2}+\sigma_{2}^{2}\right)$ & $\frac{3}{8}\left(\sigma_{1}+\sigma_{2}\right)+\frac{\left(\sigma_{2}-\sigma_{1}\right)^{2}}{8\left(\sigma_{2}+\sigma_{1}\right)}$ \\
Heteronuclear & Dimer & & \\
\hline \hline
\end{tabular}

where

$$
\alpha=\bar{r} s / 3 b
$$

is the geometric factor and $\bar{r}, s$, and $b$ are the mean radius of curvature, surface area and volume respectively, of a dimer. The geometric coefficients, $b, s$, and $\bar{r}$, for monomers, homonuclear dimers, and heteronuclear dumbbells are listed in Table I. The first- and second-order perturbation terms for the compressibility factor of a square-well dimer fluid are given by

$$
\begin{aligned}
Z_{1}^{\mathrm{dim}}= & -2 \pi \rho n^{2} \sigma^{3} \epsilon\left(I_{0}^{\mathrm{dim}}+\eta \frac{\partial I_{0}^{\mathrm{dim}}}{\partial \eta}\right) \\
Z_{2}^{\mathrm{dim}}= & -\pi \rho n^{2} \sigma^{3} \epsilon^{2}\left[\frac{f_{2}(\eta)}{f_{1}(\eta)^{2}}\left(I_{0}^{\mathrm{dim}}+\eta \frac{\partial I_{0}^{\mathrm{dim}}}{\partial \eta}\right)+\frac{f_{3}(\eta)}{f_{1}(\eta)}\right. \\
& \left.\times\left(2 \frac{\partial I_{0}^{\mathrm{dim}}}{\partial \eta}+\eta \frac{\partial^{2} I_{0}^{\mathrm{dim}}}{\partial \eta^{2}}\right)\right]
\end{aligned}
$$

where $f_{1}, f_{2}$, and $f_{3}$ are functions of $\eta$ and are defined as

$$
\begin{aligned}
f_{1}(\eta)= & 1-2 \eta+\eta^{2}+6 \alpha \eta(1-\eta)+\alpha^{2} \eta^{2}\left(9-4 \eta+\eta^{2}\right), \\
f_{2}(\eta)= & (1-\eta)^{3}\left[1-5 \eta+7 \eta^{2}-3 \eta^{3}-18 \alpha \eta^{2}(1-\eta)\right. \\
& \left.-\alpha^{2} \eta^{2}\left(9+19 \eta-5 \eta^{2}+\eta^{3}\right)\right], \\
f_{3}(\eta)= & \eta(1-\eta)^{4} .
\end{aligned}
$$

Since an analytical expression for the structure of a hard dimer fluid is not available, the integral $I_{0}^{\mathrm{dim}}$ is obtained from computer simulation on a reference hard dimer fluid. We perform molecular dynamics simulations of tangent harddimer fluids at packing fractions ranging from 0.025 to 0.475 . The radial distribution function, and upon integration the $I_{0}^{\mathrm{dim}}$, is recorded at each density. The integral $I_{0}^{\mathrm{dim}}$ is then fitted to a seventh order polynomial in $\eta$ of the form

$$
I_{0}^{\mathrm{dim}}=\sum_{k=0}^{7} c_{k} \eta^{k}
$$

This simple polynomial form facilitates easy calculation of $\partial I / \partial \eta$ and $\partial^{2} I / \partial \eta^{2}$. Table II lists the coefficients $c_{k}$ to calculate the integral $I_{0}^{\mathrm{dim}}$ using Eq. 29. It may be noted that our perturbation approach is different from Yethiraj and Hall's perturbation approach for square-well dimer fluids in that the 
TABLE II. Coefficients for the polynomial expansion of the integral $I_{0}^{\mathrm{ij}}$ $=\Sigma_{k} c_{k}^{\mathrm{ij}} \eta^{k}$.

\begin{tabular}{lccccc}
\hline \hline & & & \multicolumn{3}{c}{ Dimer-monomer mix } \\
\cline { 3 - 6 }$c_{k}$ & Monomer fluid & Dimer fluid & dim-dim & dim-mon & mon-mon \\
\hline$c_{0}$ & 0.716297 & 0.5052058 & 0.49334 & 0.68308 & 0.7584 \\
$c_{1}$ & 1.108746 & 1.074739 & 1.7335 & -0.24858 & 2.5637 \\
$c_{2}$ & 0.059607 & 1.821172 & -10.5954 & 25.0894 & -21.9074 \\
$c_{3}$ & 0.054981 & -1.462742 & 105.3595 & -211.22 & 188.53 \\
$c_{4}$ & -9.550753 & -18.976502 & -551.03 & 999.43 & -906.75 \\
$c_{5}$ & 25.31761 & 62.173311 & 1567.60 & -2695.41 & 2407.63 \\
$c_{6}$ & -53.28665 & -108.21679 & -2329.83 & 3792.53 & -3360.13 \\
$c_{7}$ & 43.11756 & 75.093454 & 1397.18 & -2163.31 & 1911.92 \\
\hline \hline
\end{tabular}

former requires a single regression term $\left(I_{0}^{\mathrm{dim}}\right)$ from simulation, while, the latter requires two regression terms $\left(A_{1}(\eta)\right.$ and $A_{2}(\eta)$ ).

\section{Square-well dimer mixtures}

A straightforward extension of the perturbation approach to square-well dimer mixtures would require an analytical expression for the radial distribution function of the reference fluid, the hard-dimer mixture, which is not currently available. Although, we could calculate the radial distribution function of the reference hard-dimer mixture using computer simulation, this would be tedious because we would have to perform computer simulations for every mixture case. Chen et al. ${ }^{48}$ devised a method to calculate a perturbation expansion for multicomponent mixtures of spherical molecules in terms of the hard-sphere pair correlation function and pair potential. However, Chen's method requires numerical integration and regression parameters. Instead, following Henderson, ${ }^{25}$ who simplified Chen's results by using simple mixing rules, we have used one-fluid mixing rules to approximate the properties of the mixture.

We extend Henderson's conformal-fluid mixing approach to square-well dimer mixtures in a manner similar to that used by Hino and Prausnitz ${ }^{26}$ for perturbed hard chain mixtures. The mixing rules are used to calculate the average perturbation terms in Eqs. 26 and 27. In effect, by using the one-fluid mixing rules we replace the mixture with a hypothetical one-component fluid that exhibits properties similar to those found for the mixture. The resulting fluid is conformal in the sense that all site-site interactions take the same average functional form.

In order to apply perturbation theory to mixtures of square-well dimers, we first identify those terms in Eqs. 26 and 27 that can be converted to a term appropriate for mixtures via mixing rules. For example, the term $\sigma^{3} n^{2} \epsilon\left(I_{0}+\eta \partial I_{0} / \partial \eta\right)$ in Eq. 26 can be calculated for mixtures using one-fluid mixing rules

$$
\begin{aligned}
{\left[n^{2} \sigma^{3} \epsilon\left(I_{0}+\eta \frac{\partial I_{0}}{\partial \eta}\right)\right]_{\operatorname{mix}}=} & n^{2} \sum_{i}^{m} \sum_{j}^{m} x_{i} x_{j} \sigma_{i j}^{3} \epsilon_{i j} \\
& \times\left(I_{0}^{i j}+\eta \frac{\partial I_{0}^{i j}}{\partial \eta}\right),
\end{aligned}
$$

where $m$ is the number of components in the dimer mixture, $x_{i}$ is the mole fraction of component $i, \sigma_{i j}=\left(\sigma_{i}+\sigma_{j}\right) / 2$ and $\epsilon_{i j}$ is defined according to the combining rules described in Eq. 2. The $I_{0}^{i j}$ are integrals of the scaled radial distribution function $\left(g_{0}^{i j}(x)\right)$ of a molecule of component $j$ about a molecule of component $i$

$$
I_{0}^{i j}=\int_{1}^{\lambda_{i}} g_{0}^{i j}(x) x^{2} d x
$$

where $x=r / \sigma_{i j}$ is the reduced unit of distance. Similarly we convert the terms $n^{2} \sigma^{3} \epsilon^{2}\left(I_{0}+\eta \partial I_{0} / \partial \eta\right)$ and $n^{2} \sigma^{3} \epsilon^{2}$ $\left(2 \partial I_{0} / \partial \eta+\eta \partial^{2} I_{0} / \partial \eta^{2}\right)$ in Eq. 27 , to the corresponding terms for square-well dimer mixtures using one-fluid mixing rules

$$
\left[n^{2} \sigma^{3} \epsilon^{2}\left(I_{0}+\eta \frac{\partial I_{0}}{\partial \eta}\right)\right]_{\text {mix }}=n^{2} \sum_{i}^{m} \sum_{j}^{m} x_{i} x_{j} \sigma_{i j}^{3} \epsilon_{i j}^{2}\left(I_{0}^{i j}+\eta \frac{\partial I_{0}^{i j}}{\partial \eta}\right)
$$

and

$$
\begin{aligned}
& {\left[n^{2} \sigma^{3} \epsilon^{2}\left(2 \frac{\partial I_{0}}{\partial \eta}+\eta \frac{\partial^{2} I_{0}}{\partial \eta^{2}}\right)\right]_{\operatorname{mix}}} \\
& \quad=n^{2} \sum_{i}^{m} \sum_{j}^{m} x_{i} x_{j} \sigma_{i j}^{3} \epsilon_{i j}^{2}\left(2 \frac{\partial I_{0}^{i j}}{\partial \eta}+\eta \frac{\partial^{2} I_{0}^{i j}}{\partial \eta^{2}}\right) .
\end{aligned}
$$

Eqs. 30, 32, and 33 require expressions for $I_{0}^{i j}$ and $g_{0}^{i j}$ of hard-dimer mixtures. However, as mentioned earlier, such expressions can not be calculated analytically. We argue that for mixtures of dimers with identical well width $\left(\lambda_{i}\right)$, we can approximate the $I_{0}^{i j}$ and its derivatives with respect to $\eta$ that appear in Eqs. 30, 32, and 33 with the equivalent expressions from a pure hard dimer fluid

$$
I_{0}^{i j} \simeq I_{0}^{\mathrm{dim}}, \quad \frac{\partial I_{0}^{i j}}{\partial \eta} \simeq \frac{\partial I_{0}^{\mathrm{dim}}}{\partial \eta}, \quad \frac{\partial^{2} I_{0}^{i j}}{\partial \eta^{2}} \simeq \frac{\partial^{2} I_{0}^{\mathrm{dim}}}{\partial \eta^{2}},
$$

where $I_{0}^{\mathrm{dim}}$ is the integral of the radial distribution function of a hard-dimer fluid at the same packing fraction as the mixture. For mixtures of square-well dimers where the two components have the same hard core diameters, the approximation is exact, since the reference fluid for such mixtures is the homonuclear hard-dimer fluid $\left(I_{0}^{i j}=I_{0}^{\mathrm{dim}}\right)$. This approximation also works well for dimer mixtures where the two components have different hard-core diameters since the integrals $I_{0}^{i j}$ are calculated by integrating the corresponding $g_{0}^{i j}$ over reduced distance $x=r / \sigma_{i j}$. In order to check the validity of the approximations in Eq. 34 , we conducted molecular dynamics simulations for hard-dimer mixtures with $\sigma_{2} / \sigma_{1}$ $=1.5$ and 2. The Appendix shows the results of these molecular dynamics simulations. We find that for the small hard-core diameter ratios studied, the approximation in Eq. 34 is indeed valid.

The final expression for the compressibility factor of a square-well dimer mixture is obtained as in Eq. 23. The reference hard-dimer mixture compressibility factor is again obtained from the scaled particle theory, 


$$
Z_{0}^{\mathrm{dim}-\mathrm{mix}}=\frac{1}{1-\eta}+\frac{3 \alpha \eta}{(1-\eta)^{2}}+\frac{3 \alpha^{2} \eta^{2}-\alpha^{2} \eta^{3}}{(1-\eta)^{3}}
$$

where the geometric factor $\alpha=\bar{r} s / 3 b$ reflects the mixture composition. The coefficients $\mathrm{b}, \mathrm{s}$, and $\bar{r}$ for a mixture are defined as

$$
b=\sum_{i}^{m} x_{i} b_{i}, \quad s=\sum_{i}^{m} x_{i} s_{i}, \quad \bar{r}=\sum_{i}^{m} x_{i} r_{i},
$$

where $x_{i}$ is the mole fraction of component $i$ and $m$ is the number of components. It may be noted that for mixtures, the term $\alpha^{2}$ in Eq. 35 is defined as

$$
\alpha^{2}=\frac{c s^{2}}{9 b^{2}},
$$

where $c=\sum_{i}{ }^{m} x_{i} r_{i}^{2}$. The first-order and second-order perturbation terms for the compressibility factor of a square-well dimer mixture then become

$$
\begin{aligned}
Z_{1}^{\mathrm{dim}-m i x}= & -2 \pi \rho\left[n^{2} \sigma^{3} \epsilon\right]_{\operatorname{mix}}\left(I_{0}^{\mathrm{dim}}+\eta \frac{\partial I_{0}^{\mathrm{dim}}}{\partial \eta}\right) \\
Z_{2}^{\mathrm{dim}-\mathrm{mix}}= & -\pi \rho\left[n^{2} \sigma^{3} \epsilon^{2}\right]_{\mathrm{mix}}\left[\frac{f_{2}(\eta)}{f_{1}(\eta)^{2}}\left(I_{0}^{\mathrm{dim}}+\eta \frac{\partial I_{0}^{\mathrm{dim}}}{\partial \eta}\right)\right. \\
& \left.+\frac{f_{3}(\eta)}{f_{1}(\eta)}\left(2 \frac{\partial I_{0}^{\mathrm{dim}}}{\partial \eta}+\eta \frac{\partial^{2} I_{0}^{\mathrm{dim}}}{\partial \eta^{2}}\right)\right]
\end{aligned}
$$

where, $f_{1}(\eta), f_{2}(\eta)$, and $f_{3}(\eta)$ are given in Eq. 28 ,

$$
\begin{aligned}
& {\left[n^{2} \sigma^{3} \epsilon\right]_{\mathrm{mix}}=n^{2} \sum_{i}^{m} \sum_{j}^{m} x_{i} x_{j} \sigma_{i j}^{3} \epsilon_{i j},} \\
& {\left[n^{2} \sigma^{3} \epsilon^{2}\right]_{\mathrm{mix}}=n^{2} \sum_{i}^{m} \sum_{j}^{m} x_{i} x_{j} \sigma_{i j}^{3} \epsilon_{i j}^{2},}
\end{aligned}
$$

and $I_{0}^{\mathrm{dim}}=\Sigma_{k}{ }^{7} c_{k} \eta^{k}$, where the coefficients $c_{k}$ are obtained from Table II.

\section{Square-well dimer-monomer mixtures}

The theory and formalism described previously for SW dimer mixtures can easily be extended to SW dimer/ monomer mixtures if we replace the factor $n^{2}$ in Eqs. 26 and 27 with $n_{i} n_{j}$ to account for the number of attractive site-site interactions per pair of molecules, where $n_{i}$ is the number of segments on molecule $i$. Using this argument, we define perturbation terms for dimer-monomer mixtures that are similar to the perturbation terms in Eqs. 30, 32, and 33

$$
\begin{aligned}
{\left[\sigma^{3} n^{2} \epsilon\right.} & \left.\left(I_{0}^{\mathrm{dim}-\mathrm{mon}}+\eta \frac{\partial I_{0}^{\mathrm{dim}-\mathrm{mon}}}{\partial \eta}\right)\right]_{\mathrm{mix}} \\
= & \sum_{i}^{m} \sum_{j}^{m} x_{i} x_{j} \sigma_{i j}^{3} n_{i} n_{j} \epsilon_{i j}\left(I_{0}^{i j}+\eta \frac{\partial I_{0}^{i j}}{\partial \eta}\right),
\end{aligned}
$$

$$
\begin{array}{r}
{\left[\sigma^{3} n^{2} \epsilon^{2}\left(I_{0}^{\mathrm{dim}-\mathrm{mon}}+\eta \frac{\partial I_{0}^{\mathrm{dim}-\mathrm{mon}}}{\partial \eta}\right)\right]_{\mathrm{mix}}} \\
=\sum_{i}^{m} \sum_{j}^{m} x_{i} x_{j} \sigma_{i j}^{3} n_{i} n_{j} \epsilon_{i j}^{2}\left(I_{0}^{i j}+\eta \frac{\partial I_{0}^{i j}}{\partial \eta}\right),
\end{array}
$$

$$
\begin{gathered}
{\left[\sigma^{3} n^{2} \epsilon^{2}\left(2 \frac{\partial I_{0}^{\mathrm{dim}-\mathrm{mon}}}{\partial \eta}+\eta \frac{\partial^{2} I_{0}^{\mathrm{dim}-\mathrm{mon}}}{\partial \eta^{2}}\right)\right]_{\mathrm{mix}}} \\
=\sum_{i}^{m} \sum_{j}^{m} x_{i} x_{j} \sigma_{i j}^{3} n_{i} n_{j} \epsilon_{i j}^{2}\left(2 \frac{\partial I_{0}^{i j}}{\partial \eta}+\eta \frac{\partial^{2} I_{0}^{i j}}{\partial \eta^{2}}\right) .
\end{gathered}
$$

In order to calculate $I_{0}^{i j}$ for the case where the monomer and dimer segments are of the same size, we performed molecular dynamics simulation on equimolar hard dimermonomer mixtures. We measured the species-species segmental radial distribution functions $\left(g_{0}^{\mathrm{dim}-\mathrm{dim}}, g_{0}^{\text {mon-mon }}\right.$, and $g_{0}^{\text {dim-mon }}$ ) and integrated over reduced distance units to obtain the respective $I_{0}^{i j}$. The integrals $I_{0}^{i j}$ were fitted to a seventh order polynomial in $\eta$ of the form

$$
I_{0}^{i j}=\int_{1}^{\lambda} g_{0}^{i j}(x) x^{2} \mathrm{~d} x=\sum_{k=0}^{7} c_{k}^{i j} \eta^{k}
$$

where $x=r / \sigma_{i j}$. Table II shows the coefficients $c_{k}$ for segmental dimer-dimer, monomer-monomer and dimermonomer interactions in a dimer-monomer mixture where the monomer and dimer segments are of the same size. When the monomer and dimer segment sizes differ, we approximate the integral $I_{0}^{i j}$ by the corresponding $I_{0}^{i j}$ for dimer/ monomer mixtures where the dimer and monomer segments are same size. In order to test the accuracy of this approximation, we once again use molecular dynamics simulations. The Appendix shows the results for $I_{0}^{i j}$ calculated from molecular dynamics simulation of hard-dimer monomer mixtures at $\eta=0.3$ where $\sigma_{d} / \sigma_{m}=1,1.5$, and 2 . We find that the values of $I_{0}^{i j}$ are relatively insensitive to $\sigma_{i j}$.

The compressibility factor of a dimer-monomer mixture is calculated according to Eq. 23. The reference hard-dimermonomer mixture compressibility factor is calculated from the scaled particle theory equation of state in Eq. 24.

$$
Z_{0}^{\mathrm{dim}-\mathrm{mon}}=\frac{1}{1-\eta}+\frac{3 \alpha \eta}{(1-\eta)^{2}}+\frac{3 \alpha^{2} \eta^{2}-\alpha^{2} \eta^{3}}{(1-\eta)^{3}}
$$

where the geometric factors $\alpha$ and $\alpha^{2}$ for a dimer-monomer mixture are calculated using Eqs. 36, 37, and Table I. The first- and second-order perturbation terms are calculated using the conformal terms defined in Eq. 40 


$$
\begin{aligned}
Z_{1}^{\mathrm{dim}-\mathrm{mon}}= & -2 \pi \rho\left[\sigma^{3} n^{2} \epsilon\left(I_{0}^{\mathrm{dim}-\mathrm{mon}}+\eta \frac{\partial I_{0}^{\mathrm{dim}-\mathrm{mon}}}{\partial \eta}\right)\right]_{\mathrm{mix}} \\
Z_{2}^{\mathrm{dim}-\mathrm{mon}}= & -\pi \rho\left\{\frac{f_{2}(\eta)}{f_{1}(\eta)^{2}}\left[\sigma^{3} n^{2} \epsilon^{2}\left(I_{0}^{\mathrm{dim}}+\eta \frac{\partial I_{0}^{\mathrm{dim}-m o n}}{\partial \eta}\right)\right]_{\text {mix }}\right. \\
& +\frac{f_{3}(\eta)}{f_{1}(\eta)}\left[\sigma ^ { 3 } n ^ { 2 } \epsilon ^ { 2 } \left(2 \frac{\partial I_{0}^{\mathrm{dim}-\mathrm{mon}}}{\partial \eta}\right.\right. \\
& \left.\left.\left.+\eta \frac{\partial^{2} I_{0}^{\mathrm{dim}-\mathrm{mon}}}{\partial \eta^{2}}\right)\right]_{\mathrm{mix}}\right\}
\end{aligned}
$$

where, $f_{1}(\eta), f_{2}(\eta)$, and $f_{3}(\eta)$ are given in Eq. 28 and $I_{0}^{\mathrm{ij}}$ $=\Sigma_{k}{ }^{7} c_{k}^{i j} \eta^{k}$, where the coefficients $c_{k}^{i j}$ for dimer-monomer mixtures are obtained from Table II.

\section{E. Heteronuclear square-well dumbbell fluids}

The reference fluid for the square-well heteronuclear dumbbell fluid is the corresponding hard-heteronuclear dumbbell fluid. To apply the conformal solution approach to calculate the perturbation terms for square-well heteronuclear dumbbell fluids, we again examine the origin of the factor $n_{i} n_{j}$ in Eq. 40 which accounts for the average number of attractive site-site interactions per pair of molecules. By definition, for every pair of heteronuclear dumbbells there are four site-site interactions: a segment 1-segment 1 interaction, a segment 2-segment 2 interaction and two segment 1-segment 2 interactions. The conformal fluid perturbation terms can be obtained by summation over all possible sitesite interaction permutations for a pair of dumbbells. The average perturbation terms for the conformal square-well heteronuclear dumbbell fluid are expressed as

$$
\begin{aligned}
& {\left[\sigma^{3} n^{2} \epsilon\left(I_{0}+\eta \frac{\partial I_{0}}{\partial \eta}\right)\right]_{\mathrm{dum}}=\sum_{i}^{2} \sum_{j}^{2} \sigma_{i j}^{3} \epsilon_{i j}\left(I_{0}^{i j}+\eta \frac{\partial I_{0}^{i j}}{\partial \eta}\right),} \\
& {\left[\sigma^{3} n^{2} \epsilon^{2}\left(I_{0}+\eta \frac{\partial I_{0}}{\partial \eta}\right)\right]_{\mathrm{dum}}=\sum_{i}^{2} \sum_{j}^{2} \sigma_{i j}^{3} \epsilon_{i j}^{2}\left(I_{0}^{i j}+\eta \frac{\partial I_{0}^{i j}}{\partial \eta}\right),} \\
& {\left[\sigma^{3} n^{2} \epsilon^{2}\left(2 \frac{\partial I_{0}}{\partial \eta}+\eta \frac{\partial^{2} I_{0}}{\partial \eta^{2}}\right)\right]_{\mathrm{dum}}} \\
& =\sum_{i}^{2} \sum_{j}^{2} \sigma_{i j}^{3} \epsilon_{i j}^{2}\left(2 \frac{\partial I_{0}^{i j}}{\partial \eta}+\eta \frac{\partial^{2} I_{0}^{i j}}{\partial \eta^{2}}\right)
\end{aligned}
$$

where the $I_{0}^{i j}$ are defined in Eq. 41. We approximate the integrals $I_{0}^{i j}$ for the reference hard heteronuclear dumbbell fluid by $I_{0}^{\mathrm{dim}}$, the integral of the radial distribution function of the corresponding homonuclear hard-dimer fluid at the same $\eta$, although, as shown in the Appendix, this approximation introduces some error. Using this approximation and the conformal fluid average parameters in Eq. 44, we can calculate the first- and second-order perturbation terms for the compressibility factor of square-well dumbbells.
The final expression for the compressibility factor of a square-well heteronuclear dumbbell fluid is obtained as in Eq. 23. The reference hard-dumbbell compressibility factor is calculated from scaled particle theory

$$
Z_{0}^{\mathrm{dum}}=\frac{p_{0}^{\mathrm{dum}}}{\rho k T}=\frac{1}{1-\eta}+\frac{3 \alpha \eta}{(1-\eta)^{2}}+\frac{3 \alpha^{2} \eta^{2}-\alpha^{2} \eta^{3}}{(1-\eta)^{3}}
$$

where the geometric parameter $\alpha$ is calculated using Eq. 36 and Table I. The first- and second-order perturbation terms for a square-well heteronuclear dumbbell fluid are

$$
\begin{aligned}
Z_{1}^{\mathrm{dum}}= & -2 \pi \rho \sum_{i}^{m} \sum_{j}^{m} \sigma_{i j}^{3} \epsilon_{i j}\left(I_{0}^{\mathrm{dim}}+\eta \frac{\partial I_{0}^{\mathrm{dim}}}{\partial \eta}\right), \\
Z_{2}^{\mathrm{dum}}= & -\pi \rho\left[\frac{f_{2}(\eta)}{f_{1}(\eta)^{2}} \sum_{i}^{m} \sum_{j}^{m} \sigma_{i j}^{3} \epsilon_{i j}^{2}\left(I_{0}^{\mathrm{dim}}+\eta \frac{\partial I_{0}^{\mathrm{dim}}}{\partial \eta}\right)\right. \\
& \left.+\frac{f_{3}(\eta)}{f_{1}(\eta)} \sum_{i} \sum_{j} \sigma_{i j}^{3} \epsilon_{i j}^{2}\left(2 \frac{\partial I_{0}^{\mathrm{dim}}}{\partial \eta}+\eta \frac{\partial^{2} I_{0}^{\mathrm{dim}}}{\partial \eta^{2}}\right)\right],
\end{aligned}
$$

where, $f_{1}(\eta), f_{2}(\eta)$, and $f_{3}(\eta)$ are given in Eq. 28 and $I_{0}$ $=\Sigma_{k}{ }^{7} c_{k} \eta^{k}$, where the parameters $c_{k}$ are obtained from Table II.

\section{COMPARISON BETWEEN THEORETICAL PREDICTIONS AND DCMD SIMULATION RESULTS}

In this section we compare our theoretical predictions for the compressibility factor to previously available MC results and to the results of our DCMD simulations. We begin by presenting the DCMD results for homonuclear square-well monomer fluids, square-well monomer mixtures, and homonuclear square-well dimer fluids in order to assess the accuracy of our DCMD calculations against available MC simulation data and against well-known theories. We then compare the DCMD simulation results to our perturbation theory results for square-well dimer mixtures, square-well dimer/monomer mixtures, and square-well heteronuclear dumbbell fluids.

\section{A. Square-well homonuclear monomer fluid}

Figure 3 compares the results of our DCMD simulation (indicated by filled symbols) of square-well monomers to theoretical predictions at reduced temperatures $T^{*} \equiv k T / \epsilon$ $=1.5$ and 3 . We show the predictions of three equations of state: Yethiraj and Hall's ${ }^{17}$ perturbation theory, Bokis et al.' ${ }^{49}$ local composition model and Chang and Sandler's ${ }^{22}$ analytical expression from perturbation theory. The results of MC simulation by Tavares et $a .^{21}$ are also shown (indicated by open symbols). The MC and DCMD results are in very close agreement with each other. All three equations of state are very accurate in predicting the compressibility factors of square-well monomer fluids over the entire range of densities and temperatures studied, with Chang and Sandler's expression being the most accurate. It may be noted that this list of 


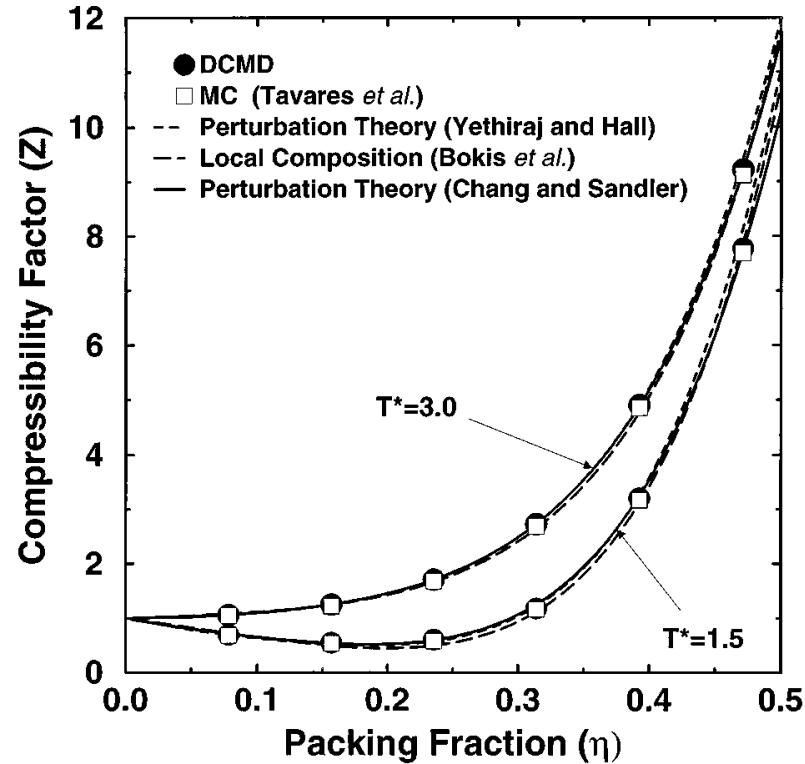

FIG. 3. Comparison of DCMD and Monte Carlo compressibility factors with theoretical predictions for square-well homonuclear monomers at $T^{*}$ $=1.5$ and $T^{*}=3$. Filled circles represent our DCMD simulation results, open squares are the results of MC simulation of Tavares et al. (Ref. 21). The dotted lines represent the predictions of Yethiraj and Hall's (Ref. 17) perturbation theory, dashed lines are the predictions of the local composition model of Bokis et al. (Ref. 49), and the solid lines are the predictions of Chang and Sandler's (Ref. 22) perturbation theory.

equations of state for square-well monomer fluids is by no means exhaustive. We have shown only a selected number of equations of state, since our only objective here is to check the accuracy of our DCMD technique.

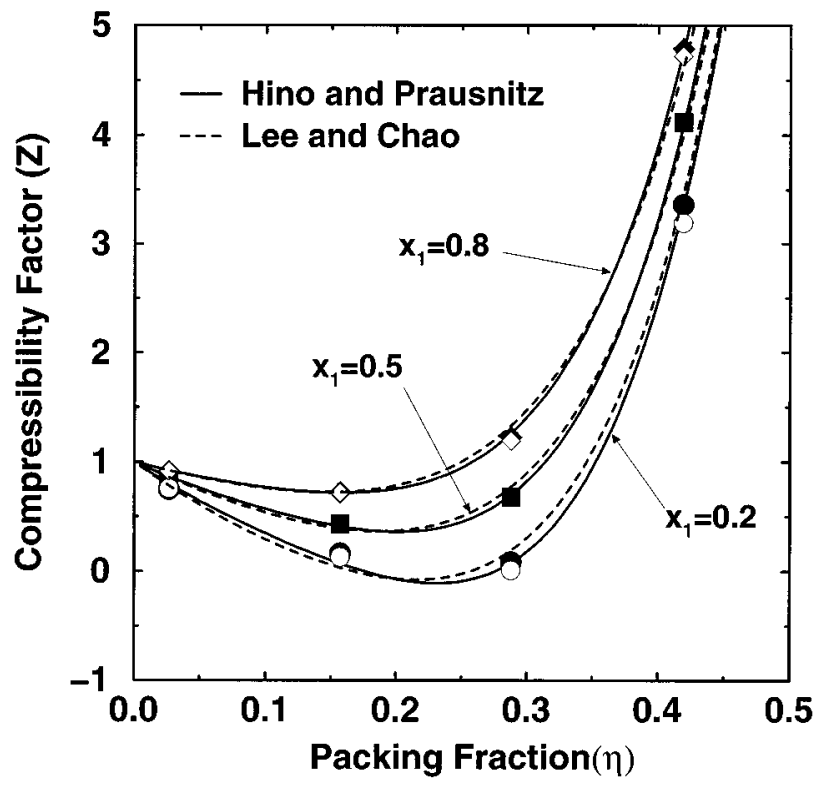

FIG. 4. Comparison of DCMD and Monte Carlo compressibility factors for square-well monomer mixtures with theoretical predictions. Three different compositions are studied for mixtures where $\sigma_{2} / \sigma_{1}=1.0, \epsilon_{22} / \epsilon_{11}=2$ and $T^{*}=2$. Filled symbols represent our DCMD simulation results, open symbols are MC simulation results of Lee and Chao (Ref. 11), dotted lines are the predictions of Lee and Chao's local composition model, and solid lines are the predictions of Hino and Prausnitz's perturbation theory (Ref. 26).

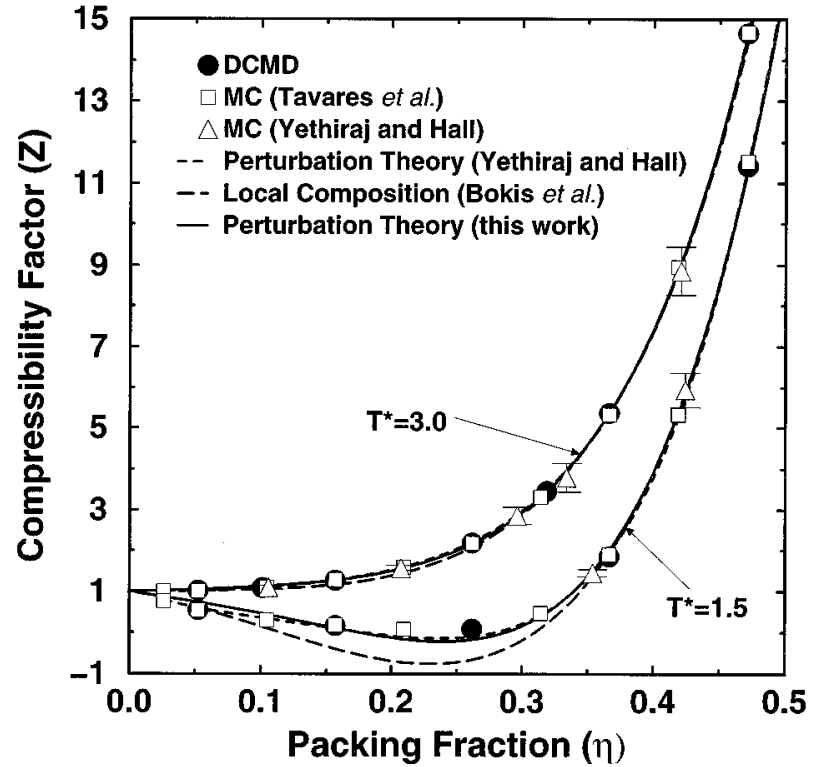

FIG. 5. Comparison of DCMD and Monte Carlo compressibility factors for square-well homonuclear dimer fluids at $T^{*}=1.5$ and $T^{*}=3$. Filled circles represent our DCMD simulation results, open squares are the MC simulation results of Tavares et al. (Ref. 21) and the open triangles are the MC simulation results of Yethiraj and Hall (Ref. 17). Dotted lines represent the predictions of Yethiraj and Hall's (Ref. 17) perturbation theory, dashed lines are the predictions of Bokis et al.'s (Ref. 49) and the solid lines are the predictions of our perturbation theory.

\section{B. Square-well monomer mixtures}

Figure 4 shows the compressibility factor vs. packing fraction for square-well monomer mixtures at three different compositions: $x_{1}=0.2,0.5$, and 0.8 , where $\sigma_{2} / \sigma_{1}=1.0$, $\epsilon_{2} / \epsilon_{1}=2$, and $T^{*} \equiv k T / \epsilon_{1}=2$. The filled symbols are the results of our DCMD simulation, the open symbols are the MC simulation results of Lee and Chao, ${ }^{50}$ the solid lines represent the predictions of Hino and Prausnitz's perturbation theory, ${ }^{26}$ and the dotted lines represent the predictions of Lee and Chao's local composition model. The DCMD compressibility factor and the MC simulation results agree well at low and moderate densities. At higher densities, the compressibility factor values obtained from DCMD simulation are slightly larger than the MC compressibility factors, although the results may be within simulation uncertainties (Lee and $\mathrm{Chao}^{50}$ do not report error bars for their MC simulation results). Both the perturbation theory and the local composition model are found to accurately predict the compressibility factor of square-well monomer mixtures.

\section{Square-well homonuclear dimer fluid}

Figure 5 shows the compressibility factor vs. packing fraction for square-well homonuclear dimer fluids at two temperatures: $T^{*}=1.5$ and 3 . We compare the results of our DCMD simulation ( $)$ and previous MC simulation by Tavares et al. ${ }^{21}(\square)$ and by Yethiraj and $\operatorname{Hall}^{17}(\triangle)$, with theoretical predictions based on the perturbation theory of Yethiraj and Hall, the local composition model of Bokis et al. ${ }^{49}$ and the perturbation theory presented in this work (Eqs. 24, 26, and 27). Once again we observe excellent 
TABLE III. The DCMD simulation values of compressibility factors for binary square-well homonuclear dimer mixtures at $T^{*}=2$ and $T^{*}=3$. The value in parentheses represents one standard deviation in the last two significant digits.

\begin{tabular}{|c|c|c|c|c|c|c|c|}
\hline \multirow[b]{2}{*}{$\sigma_{2} / \sigma_{1}$} & \multirow[b]{2}{*}{$\epsilon_{22} / \epsilon_{11}$} & \multirow[b]{2}{*}{$x_{1}$} & \multirow[b]{2}{*}{$\eta$} & \multicolumn{2}{|c|}{$T^{*}=2$} & \multicolumn{2}{|c|}{$T^{*}=3$} \\
\hline & & & & $\mathrm{Z}(\mathrm{MD})$ & Z(Theory) & $\mathrm{Z}(\mathrm{MD})$ & Z(Theory) \\
\hline \multirow[t]{5}{*}{1} & 1 & 0.5 & 0.09 & $0.7387(12)$ & 0.8289 & $1.0726(05)$ & 1.1137 \\
\hline & & & 0.18 & $0.7065(20)$ & 0.6916 & $1.3869(31)$ & 1.3905 \\
\hline & & & 0.27 & 1.1398 (59) & 1.1109 & $2.3259(89)$ & 2.3239 \\
\hline & & & 0.36 & $3.2874(32)$ & 3.4409 & $5.0343(11)$ & 5.0873 \\
\hline & & & 0.45 & $10.2727(29)$ & 10.3295 & $11.9926(10)$ & 12.0298 \\
\hline \multirow[t]{5}{*}{1} & 0.5 & 0.5 & 0.09 & $1.0049(32)$ & 1.0603 & $1.2389(10)$ & 1.2643 \\
\hline & & & 0.18 & $1.2582(21)$ & 1.2609 & $1.7691(29)$ & 1.7713 \\
\hline & & & 0.27 & 2.1058 (89) & 2.0998 & $3.0024(72)$ & 2.9906 \\
\hline & & & 0.36 & $4.7318(74)$ & 4.7833 & $5.9996(43)$ & 5.9930 \\
\hline & & & 0.45 & $11.6957(65)$ & 11.7156 & $12.9556(67)$ & 12.9633 \\
\hline \multirow[t]{5}{*}{1} & 1.5 & 0.2 & 0.09 & $0.2593(23)$ & 0.4827 & $0.8084(20)$ & 0.8908 \\
\hline & & & 0.18 & $0.0497(77)$ & -0.1259 & $0.8507(22)$ & 0.8426 \\
\hline & & & 0.27 & $-0.0390(63)$ & -0.2918 & $1.3920(40)$ & 1.3724 \\
\hline & & & 0.36 & $1.3032(49)$ & 1.5393 & $3.6844(84)$ & 3.7958 \\
\hline & & & 0.45 & $8.2798(47)$ & 8.3605 & $10.6246(92)$ & 10.6962 \\
\hline \multirow[t]{5}{*}{1} & 1.5 & 0.5 & 0.09 & $0.4566(08)$ & 0.6200 & $0.9149(20)$ & 0.9790 \\
\hline & & & 0.18 & $0.2889(66)$ & 0.1962 & $1.0597(07)$ & 1.0585 \\
\hline & & & 0.27 & $0.3992(20)$ & 0.2599 & $1.7544(21)$ & 1.7470 \\
\hline & & & 0.36 & 2.1032 (99) & 2.2870 & 4.2087 (88) & 4.3042 \\
\hline & & & 0.45 & $9.0711(79)$ & 9.1351 & $11.1888(63)$ & 11.2212 \\
\hline \multirow[t]{5}{*}{1} & 1.5 & 0.8 & 0.09 & $0.6331(11)$ & 0.7483 & $1.0108(05)$ & 1.0617 \\
\hline & & & 0.18 & $0.5386(30)$ & 0.4997 & 1.2581 (19) & 1.2619 \\
\hline & & & 0.27 & $0.8421(22)$ & 0.7808 & $2.1104(81)$ & 2.1002 \\
\hline & & & 0.36 & $2.8325(90)$ & 2.9932 & $4.7245(48)$ & 4.7837 \\
\hline & & & 0.45 & $9.8029(11)$ & 9.8662 & $11.6798(94)$ & 11.7163 \\
\hline \multirow[t]{5}{*}{2} & 1 & 0.5 & 0.09 & $0.7299(13)$ & 0.8266 & 1.0417 (17) & 1.0804 \\
\hline & & & 0.18 & $0.6478(63)$ & 0.6635 & $1.2636(29)$ & 1.2780 \\
\hline & & & 0.27 & $0.8659(53)$ & 0.9364 & $1.9467(49)$ & 1.9938 \\
\hline & & & 0.36 & $2.3539(57)$ & 2.7552 & $3.9624(58)$ & 4.1861 \\
\hline & & & 0.45 & $7.8323(95)$ & 8.2830 & $9.2522(91)$ & 9.7605 \\
\hline \multirow[t]{5}{*}{2} & 0.5 & 0.5 & 0.09 & $1.0690(06)$ & 1.1140 & $1.2480(31)$ & 1.2666 \\
\hline & & & 0.18 & $1.3219(11)$ & 1.3633 & 1.7335 (19) & 1.7453 \\
\hline & & & 0.27 & $2.0593(55)$ & 2.1430 & $2.7746(25)$ & 2.8080 \\
\hline & & & 0.36 & $4.1123(66)$ & 4.3885 & $5.1405(32)$ & 5.2901 \\
\hline & & & 0.45 & $9.3065(28)$ & 9.9690 & $10.7045(49)$ & 10.8983 \\
\hline \multirow[t]{5}{*}{2} & 1.5 & 0.5 & 0.09 & $0.3605(11)$ & 0.5490 & $0.8373(17)$ & 0.9031 \\
\hline & & & 0.18 & $0.1269(23)$ & 0.0195 & $0.8500(43)$ & 0.8474 \\
\hline & & & 0.27 & $-0.0451(84)$ & -0.1551 & $1.2160(32)$ & 1.2521 \\
\hline & & & 0.36 & $0.7641(51)$ & 1.2820 & $2.8798(40)$ & 3.1823 \\
\hline & & & 0.45 & $5.8158(95)$ & 6.7580 & $8.1079(87)$ & 8.7242 \\
\hline
\end{tabular}

agreement between DCMD and MC simulation results. It may be noted that for $T^{*}=1.5$, the simulation results at low to intermediate densities are suspect since these state points are probably in the two phase region. ${ }^{17}$ Out of the three equations of state presented in Fig. 5, Yethiraj and Hall's perturbation theory most accurately predicts the compressibility factors of a square-well dimer fluid. This is because Yethiraj and Hall obtained the expressions for first- and second-order perturbation terms for free energy by separately fitting them to simulation results while our perturbation theory uses a single regression expression for the integral of the radial distribution function.

\section{Square-well dimer mixtures}

Having established the accuracy of our molecular dynamics simulation technique and of our perturbation theory

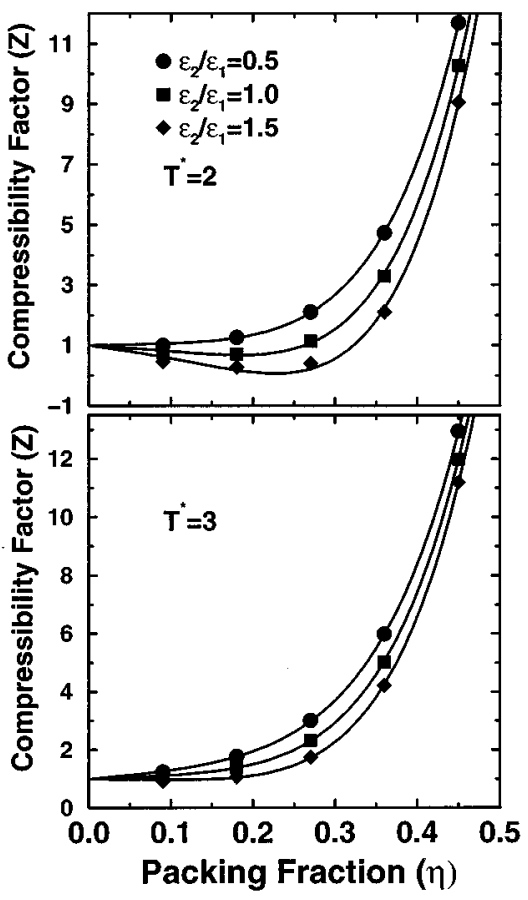

FIG. 6. Comparison of DCMD simulation results and predictions of perturbation theory for square-well dimer mixtures at $T^{*}=2$ and $T^{*}=3$ where $\sigma_{2} / \sigma_{1}=1$ and the composition of all mixtures is $x_{1}=0.5$. Results are shown for three different well depth ratios ranging from $\epsilon_{2} / \epsilon_{1}=0.5$ to $\epsilon_{2} / \epsilon_{1}$ $=1.5$.

for square-well homonuclear dimer fluids, we compare the predictions of our perturbation theory for square-well dimer mixtures with DCMD simulation results. Table III summarizes the simulation results for all the dimer mixture cases considered at reduced temperatures $T^{*} \equiv k T / \epsilon_{1}=2$ and 3 . The compressibility factor predictions of our perturbation theory are also shown.

Figures 6-8 show the DCMD simulation results and theoretical predictions for the compressibility factors of squarewell dimer mixtures as a function of the packing fraction. All mixture results are presented at two reduced temperatures: $T^{*}=2$ and $T^{*}=3$. In Fig. $6, \sigma_{2} / \sigma_{1}=1$ and $x_{1}=0.5$, while the relative well depth $\epsilon_{2} / \epsilon_{1}$ is varied from 0.5 to 1.5 . In Fig. $7, \sigma_{2} / \sigma_{1}=2$ and $x_{1}=0.5$, while the relative well depth $\epsilon_{2} / \epsilon_{1}$ is again varied from 0.5 to 1.5 . Figure 8 shows the compressibility factors of square-well dimer mixtures at three different compositions: $x_{1}=0.2,0.5$, and 0.8 , for $\sigma_{2} / \sigma_{1}=1$ and $\epsilon_{2} / \epsilon_{1}=1.5$. In all figures, the solid lines represent the predictions of the perturbation theory (Eqs. 35 and 38). The perturbation theory accurately predicts the compressibility factors of square-well dimer mixtures over the entire range of densities and compositions studied, except as noted below.

In general, the predictions of the perturbation theory are more accurate at higher densities than at lower densities. This is because the effect of attractive attraction on the local structure of the fluid is most significant at lower densities. In other words, the structure of the square-well fluid deviates 


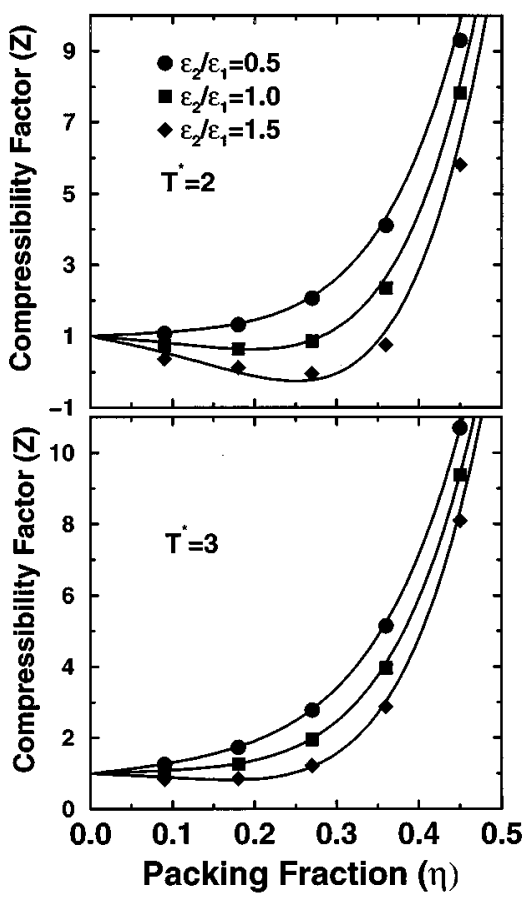

FIG. 7. Comparison of DCMD simulation results and predictions of perturbation theory for square-well dimer mixtures at $T^{*}=2$ and $T^{*}=3$ where $\sigma_{2} / \sigma_{1}=2$ and the composition of all mixtures is $x_{1}=0.5$. Results are shown for three different well depth ratios ranging from $\epsilon_{2} / \epsilon_{1}=0.5$ to $\epsilon_{2} / \epsilon_{1}$ $=1.5$.

most significantly from the corresponding hard-sphere fluid at low densities. As density increases, packing effects (entropic hard-core repulsion) begin to dominate and the attractive interactions play a lesser role in altering the local composition of the fluid. This trend can be observed in Fig. 6 and Fig. 7, where, irrespective of the relative well-depth ratios of the two component, the compressibility factor curves begin to converge at high density. Thus, expressing the properties of a square-well fluid in terms of the structure of the corresponding reference hard-sphere fluid at the same density (the underlying premise of perturbation theory), results in largest errors at low densities.

On similar lines, the accuracy of perturbation theory also improves with increasing temperature since the attractive interaction effects (potential energy) are dominated by the kinetic energy of the particle. The attractive interaction effects at low densities and low temperatures $\left(T^{*}=2\right)$ are observed in Figs. 7 and 8 in the form of the deviation between simulation results and perturbation theory predictions. For the cases where the second component has a larger well-depth $\left(\epsilon_{2} / \epsilon_{1}=1.5\right)$, the perturbation theory predictions become less accurate, especially at low densities. Since the reduced temperature $T^{*}$ is defined as $k T / \epsilon_{1}$, a deeper well for the second component, corresponds to a lower effective system temperature. Additionally, the simulation data in these cases is also suspect since these states may difference in the hardcore diameters of the two components. be in the two phase region. We also note that the accuracy of the perturbation theory deteriorates with increasing

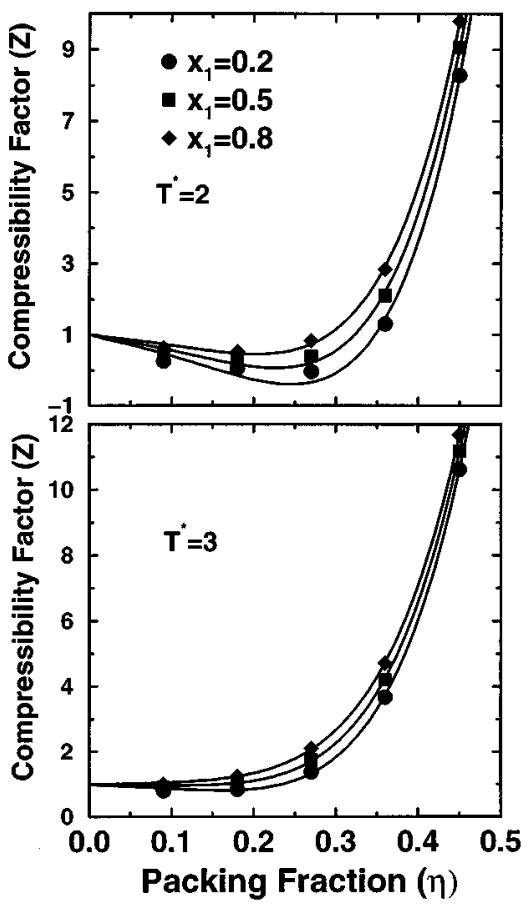

FIG. 8. Comparison of DCMD simulation results and predictions of perturbation theory and for square-well dimer mixtures at $T^{*}=2$ and 3 where $\sigma_{2} / \sigma_{1}=1$ and $\epsilon_{2} / \epsilon_{1}=1.5$. Results are shown for three different mixture compositions $x_{1}=0.2,0.5$, and 0.8 .

The perturbation theory overpredicts the compressibility factor at high densities when $\sigma_{2} / \sigma_{1}=2$ (Fig. 7). This is a limitation of the mixing rule approach since with increasing disparity between the two components, the conformal fluid mixing approximation introduces larger errors.

\section{E. Square-well dimer-monomer mixtures}

We now compare the predictions of the perturbation theory for square-well dimer-monomer mixtures (Eqs. 42 and 43) with the results of our DCMD and previous MC simulation. Table IV summarizes the simulation results for all square-well dimer-monomer mixtures studied using DCMD simulation. Figures 9 and 10 compare theoretical predictions to simulation results for the compressibility factors of square-well dimer-monomer mixtures where $\sigma_{d} / \sigma_{m}$ $=1, x_{d}=0.333$ (Fig. 9); and $\sigma_{d} / \sigma_{m}=0.5, x_{d}=0.333$ (Fig. 10). For each case, we consider three relative well-depth ratios: $\epsilon_{d} / \epsilon_{m}=0.5,1.0,1.5$ and two reduced temperatures: $T^{*}=2$ and 3 . The filled symbols represent the results of our DCMD simulation and the open symbols are the results of MC simulation. ${ }^{51}$ The solid lines represent the predictions of our perturbation theory. Once again the DCMD simulation data agrees very well with MC simulation data, reaffirming the accuracy of our DCMD simulation technique. The predictions of the perturbation theory also agree very well with the DCMD and MC simulation results at intermediate to high 
TABLE IV. DCMD simulation values of compressibility factors for square-well dimer-monomer mixtures at $T^{*}=2$ and $T^{*}=3$. The value in parentheses represents one standard deviation in the last two significant digits.

\begin{tabular}{|c|c|c|c|c|c|c|c|c|}
\hline \multirow[b]{2}{*}{$\sigma_{d} / \sigma_{m}$} & \multirow[b]{2}{*}{$\epsilon_{d} / \epsilon_{m}$} & \multirow[b]{2}{*}{$x_{d}^{\text {site }}$} & \multirow[b]{2}{*}{$x_{d}$} & \multirow[b]{2}{*}{$\eta$} & \multirow{2}{*}{$\begin{array}{l}T^{*}=2 \\
\mathrm{Z}(\mathrm{MD})\end{array}$} & \multicolumn{2}{|c|}{$T^{*}=3$} & \multirow[b]{2}{*}{ Z(Theory) } \\
\hline & & & & & & Z(Theory) & $\mathrm{Z}(\mathrm{MD})$ & \\
\hline \multirow[t]{5}{*}{1} & 1 & 0.5 & 0.33 & 0.09 & 0.8329 (13) & 0.8597 & $1.0782(08)$ & 1.0889 \\
\hline & & & & 0.18 & 0.8653 (17) & 0.8394 & $1.3626(10)$ & 1.3542 \\
\hline & & & & 0.27 & $1.3070(32)$ & 1.2957 & $2.1403(28)$ & 2.1376 \\
\hline & & & & 0.36 & $3.0745(76)$ & 3.1213 & $4.2189(14)$ & 4.2210 \\
\hline & & & & 0.45 & $8.0808(60)$ & 8.0372 & $9.1673(22)$ & 9.1446 \\
\hline \multirow[t]{5}{*}{1} & 0.5 & 0.5 & 0.33 & 0.09 & $1.0189(10)$ & 1.0324 & $1.1964(05)$ & 1.2007 \\
\hline & & & & 0.18 & $1.2423(27)$ & 1.2363 & $1.6238(08)$ & 1.6195 \\
\hline & & & & 0.27 & $1.9590(08)$ & 1.9576 & $2.5924(19)$ & 2.5851 \\
\hline & & & & 0.36 & $3.9887(12)$ & 3.9999 & $4.8305(56)$ & 4.8161 \\
\hline & & & & 0.45 & $8.9886(69)$ & 8.9339 & $9.7767(68)$ & 9.7516 \\
\hline \multirow[t]{5}{*}{1} & 1.5 & 0.5 & 0.33 & 0.09 & $0.6484(12)$ & 0.7045 & $0.9690(06)$ & 0.9897 \\
\hline & & & & 0.18 & $0.5662(12)$ & 0.4966 & $1.1431(21)$ & 1.1248 \\
\hline & & & & 0.27 & $0.8016(03)$ & 0.7311 & $1.7756(25)$ & 1.7535 \\
\hline & & & & 0.36 & $2.3136(34)$ & 2.3733 & $3.7006(56)$ & 3.7104 \\
\hline & & & & 0.45 & $7.3189(91)$ & 7.2679 & $8.6533(44)$ & 8.6207 \\
\hline \multirow[t]{5}{*}{2} & 1 & 0.5 & 0.33 & 0.09 & 0.8477 (14) & 0.8901 & $1.0629(02)$ & 1.0805 \\
\hline & & & & 0.18 & $0.8283(16)$ & 0.8612 & $1.2663(13)$ & 1.2907 \\
\hline & & & & 0.27 & $1.0524(59)$ & 1.1938 & $1.8090(14)$ & 1.9010 \\
\hline & & & & 0.36 & $2.1903(44)$ & 2.6149 & $3.2999(37)$ & 3.5480 \\
\hline & & & & 0.45 & $5.8212(31)$ & 6.5358 & $7.0173(21)$ & 7.4909 \\
\hline \multirow[t]{5}{*}{2} & 0.5 & 0.5 & 0.33 & 0.09 & $1.0773(14)$ & 1.0969 & $1.2042(02)$ & 1.2135 \\
\hline & & & & 0.18 & $1.2958(19)$ & 1.3359 & $1.5869(13)$ & 1.6072 \\
\hline & & & & 0.27 & $1.8712(37)$ & 1.9853 & $2.3739(14)$ & 2.4364 \\
\hline & & & & 0.36 & $3.3831(55)$ & 3.6681 & $4.0946(37)$ & 4.2632 \\
\hline & & & & 0.45 & 7.0688 (19) & 7.6237 & 7.8709 (21) & 8.2277 \\
\hline \multirow[t]{5}{*}{2} & 1.5 & 0.5 & 0.33 & 0.09 & $0.6111(24)$ & 0.6904 & $0.9239(09)$ & 0.9542 \\
\hline & & & & 0.18 & $0.4579(15)$ & 0.4260 & $0.9852(26)$ & 1.0007 \\
\hline & & & & 0.27 & $0.4035(41)$ & 0.4832 & $1.3162(41)$ & 1.4167 \\
\hline & & & & 0.36 & $1.1193(84)$ & 1.6757 & $2.5769(65)$ & 2.9033 \\
\hline & & & & 0.45 & $4.6958(76)$ & 5.5543 & $6.2581(83)$ & 6.8212 \\
\hline \multirow[t]{5}{*}{0.5} & 1 & 0.5 & 0.33 & 0.09 & $0.8171(22)$ & 0.8356 & $1.0506(08)$ & 1.0536 \\
\hline & & & & 0.18 & $0.8037(14)$ & 0.7777 & $1.2746(13)$ & 1.2542 \\
\hline & & & & 0.27 & $1.1278(04)$ & 1.1322 & 1.8999 (49) & 1.8918 \\
\hline & & & & 0.36 & $2.4882(20)$ & 2.6333 & $3.5288(66)$ & 3.6068 \\
\hline & & & & 0.45 & $6.3035(29)$ & 6.6726 & $7.4035(56)$ & 7.6394 \\
\hline \multirow[t]{5}{*}{0.5} & 0.5 & 0.5 & 0.33 & 0.09 & $0.9283(05)$ & 0.9292 & $1.1163(08)$ & 1.1138 \\
\hline & & & & 0.18 & $1.0304(20)$ & 0.9875 & $1.4249(13)$ & 1.3942 \\
\hline & & & & 0.27 & 1.5009 (17) & 1.4747 & $2.1552(49)$ & 2.1238 \\
\hline & & & & 0.36 & $3.0318(25)$ & 3.0823 & $3.9065(66)$ & 3.9117 \\
\hline & & & & 0.45 & $6.9397(22)$ & 7.1225 & $7.8429(56)$ & 7.9460 \\
\hline \multirow[t]{5}{*}{0.5} & 1.5 & 0.5 & 0.33 & 0.09 & $0.7089(11)$ & 0.7571 & $0.9914(09)$ & 1.0036 \\
\hline & & & & 0.18 & $0.6182(14)$ & 0.6080 & $1.1496(15)$ & 1.1409 \\
\hline & & & & 0.27 & $0.8544(46)$ & 0.8593 & $1.6924(31)$ & 1.7058 \\
\hline & & & & 0.36 & $2.0732(48)$ & 2.2764 & $3.2337(32)$ & 3.3627 \\
\hline & & & & 0.45 & $5.7901(59)$ & 6.3145 & $7.0351(67)$ & 7.3936 \\
\hline
\end{tabular}

densities. At very high densities for the case where $\sigma_{d} / \sigma_{m}$ $=0.5$ and $\epsilon_{d} / \epsilon_{m}=1.5$ (Fig. 10) the perturbation theory overpredicts the compressibility factor.

\section{F. Square-well heteronuclear dumbbell fluids}

Table V summarizes the simulation results for the square-well heteronuclear dumbbell fluids studied via DCMD simulation. Figures 11 and 12 compare the compressibility factor predictions of the perturbation theory for square-well heteronuclear dumbbell fluids and our DCMD simulation results. For each case, we consider three relative well-depth ratios: $\epsilon_{2} / \epsilon_{1}=0.5,1.0,1.5$, and two reduced temperatures: $T^{*}=2$ and 3. In Fig. 11 the two components have the same hard-core diameters, $\sigma_{2} / \sigma_{1}=1$, while in Fig. 12, the ratio of the hard-core diameters is $\sigma_{2} / \sigma_{1}=2$. The predictions of perturbation theory agree very well with DCMD simulation results at intermediate to high densities for the range of relative well depths and reduced temperatures studied. Again the perturbation theory slightly overestimates the compressibility factors at very high densities for the case when $\sigma_{2} / \sigma_{1}=2.0$ and $\epsilon_{2} / \epsilon_{1}=1.5$ (Fig. 12). Additionally, the perturbation theory is less accurate at low densities and 


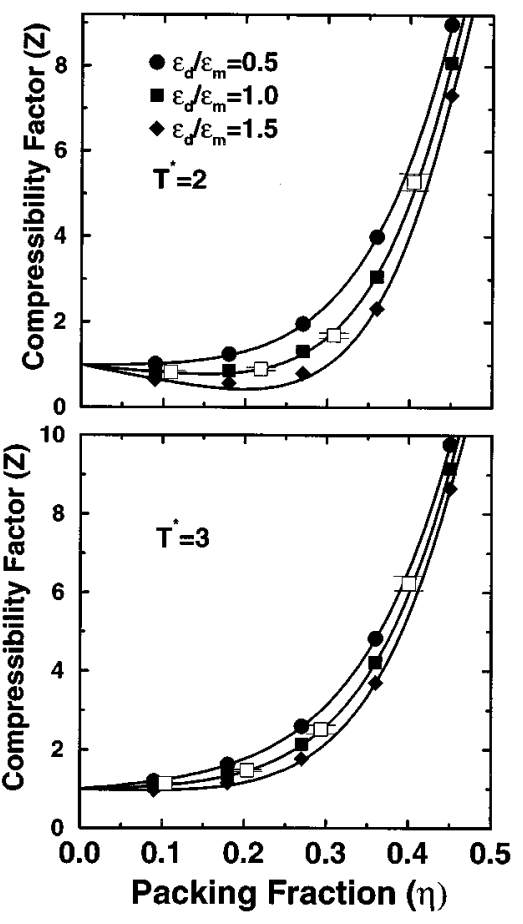

FIG. 9. Comparison of DCMD simulation results and predictions of perturbation theory for square-well dimer-monomer mixtures at $T^{*}=2$ and 3 where $\sigma_{d} / \sigma_{m}=1$ and $x_{d}=0.333$. The filled symbols represent the results of our DCMD simulation, the open symbols are the results of MC simulation (Ref. 51), and the solid lines depict the predictions of the perturbation theory.

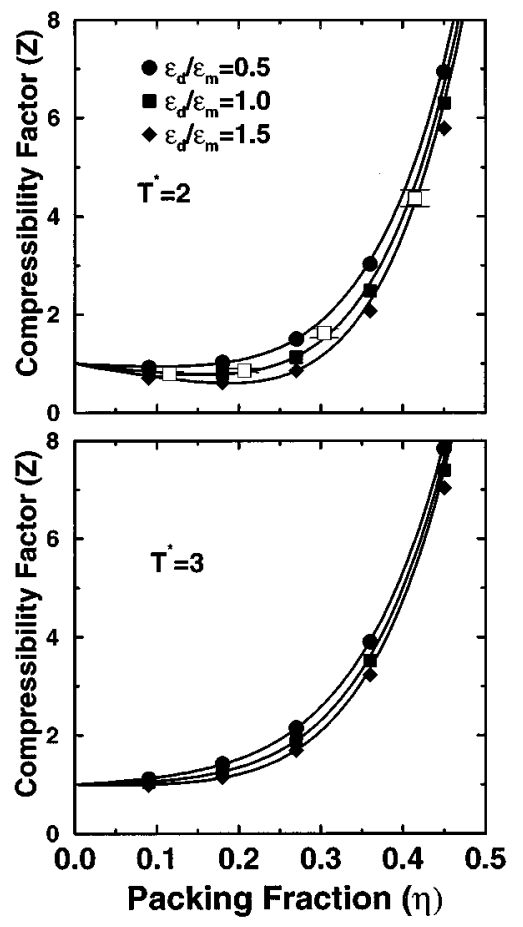

FIG. 10. Comparison of DCMD simulation results and predictions of perturbation theory for square-well dimer-monomer mixtures at $T^{*}=2$ and 3 where $\sigma_{d} / \sigma_{m}=0.5$ and $x_{d}=0.333$. The filled symbols represent the results of our DCMD simulation, the open symbols are the results of MC simulation (Ref. 51).

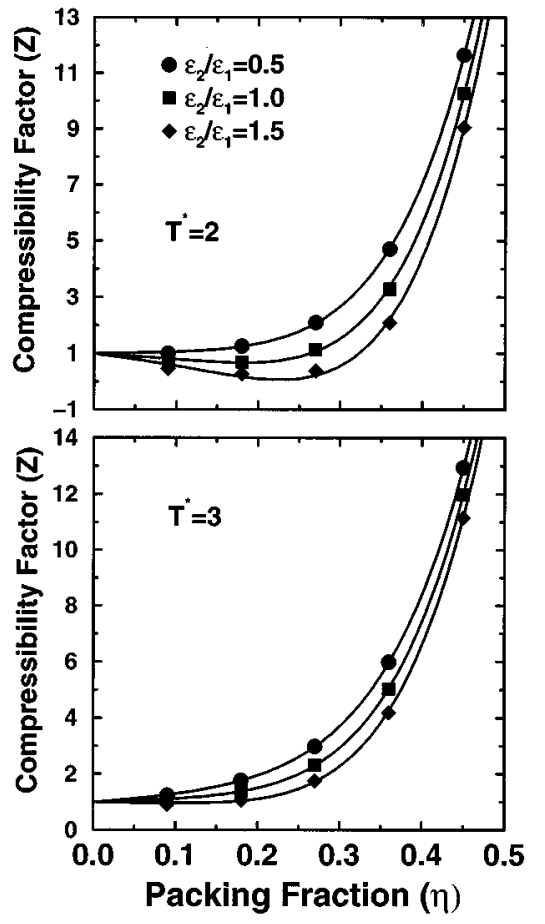

FIG. 11. Comparison of DCMD simulation results and predictions of perturbation theory for square-well heteronuclear dumbbell fluids at $T^{*}=2$ and 3 where $\sigma_{2} / \sigma_{1}=1$. Results are shown for three different well depth ratios ranging from $\epsilon_{2} / \epsilon_{1}=0.5$ to $\epsilon_{2} / \epsilon_{1}=1.5$.

TABLE V. The DCMD simulation values of compressibility factors for square-well heteronuclear dumbbell fluids at $T^{*}=2$ and $T^{*}=3$. The value in parentheses represents one standard deviation in the last two significant digits.

\begin{tabular}{|c|c|c|c|c|c|c|}
\hline \multirow[b]{2}{*}{$\sigma_{2} / \sigma_{1}$} & \multirow[b]{2}{*}{$\epsilon_{22} / \epsilon_{11}$} & \multirow[b]{2}{*}{$\eta$} & \multicolumn{2}{|c|}{$T^{*}=2$} & \multicolumn{2}{|c|}{$T^{*}=3$} \\
\hline & & & Z(MD) & Z(Theory) & $\mathrm{Z}(\mathrm{MD})$ & Z(Theory) \\
\hline \multirow[t]{5}{*}{1} & 0.5 & 0.09 & $1.0077(02)$ & 1.0600 & 1.2404 (13) & 1.2642 \\
\hline & & 0.18 & $1.2511(15)$ & 1.2608 & $1.7657(42)$ & 1.7712 \\
\hline & & 0.27 & $2.1005(83)$ & 2.1006 & $2.9950(77)$ & 2.9910 \\
\hline & & 0.36 & $4.7106(12)$ & 4.7850 & $5.9881(69)$ & 5.9938 \\
\hline & & 0.45 & $11.6619(24)$ & 11.7174 & $12.9468(74)$ & 12.9641 \\
\hline \multirow[t]{5}{*}{1} & 1.5 & 0.09 & $0.4662(30)$ & 0.6193 & $0.9141(09)$ & 0.9787 \\
\hline & & 0.18 & $0.2743(37)$ & 0.1958 & $1.0569(15)$ & 1.0583 \\
\hline & & 0.27 & $0.3867(69)$ & 0.2621 & $1.7507(26)$ & 1.7480 \\
\hline & & 0.36 & $2.0903(81)$ & 2.2919 & $4.2033(54)$ & 4.3063 \\
\hline & & 0.45 & 9.0541 (97) & 9.1401 & $11.1624(66)$ & 11.2234 \\
\hline \multirow[t]{5}{*}{2} & 1 & 0.09 & $0.7085(03)$ & 0.8402 & 1.0287 (19) & 1.0939 \\
\hline & & 0.18 & $0.6340(58)$ & 0.6905 & $1.2582(09)$ & 1.3055 \\
\hline & & 0.27 & $0.9194(45)$ & 0.9784 & $1.9864(42)$ & 2.0357 \\
\hline & & 0.36 & 2.4987 (43) & 2.8073 & $4.0789(30)$ & 4.2366 \\
\hline & & 0.45 & $7.6730(91)$ & 8.3207 & $9.4136(91)$ & 9.7960 \\
\hline \multirow[t]{5}{*}{2} & 0.5 & 0.09 & $1.0804(11)$ & 1.1275 & $1.2576(14)$ & 1.2800 \\
\hline & & 0.18 & $1.3699(38)$ & 1.3908 & $1.7640(04)$ & 1.7731 \\
\hline & & 0.27 & $2.1582(98)$ & 2.1849 & $2.8496(75)$ & 2.8499 \\
\hline & & 0.36 & $4.2707(56)$ & 4.4389 & $5.2813(32)$ & 5.3399 \\
\hline & & 0.45 & 9.5277 (84) & 10.0043 & $10.6791(73)$ & 10.9327 \\
\hline \multirow[t]{5}{*}{2} & 1.5 & 0.09 & $0.2784(54)$ & 0.5628 & $0.7987(24)$ & 0.9166 \\
\hline & & 0.18 & $0.0431(86)$ & 0.0456 & $0.7988(29)$ & 0.8746 \\
\hline & & 0.27 & $-0.0777(57)$ & -0.1131 & $1.2022(24)$ & 1.2941 \\
\hline & & 0.36 & $0.8955(93)$ & 1.3365 & 2.9808 (93) & 3.2339 \\
\hline & & 0.45 & 6.0546 (99) & 6.7991 & $8.2918(20)$ & 8.7611 \\
\hline
\end{tabular}



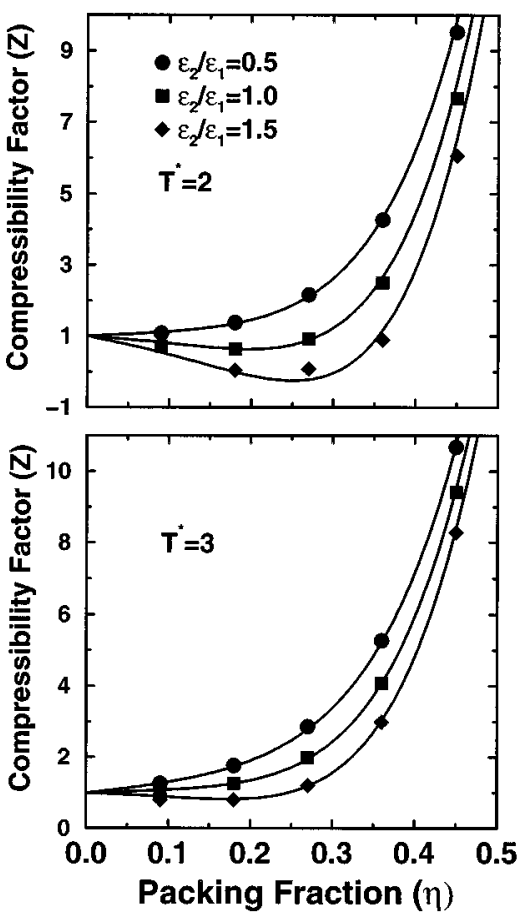

FIG. 12. Comparison of DCMD simulation results and predictions of perturbation theory for square-well heteronuclear dumbbell fluids at $T^{*}=2$ and 3 where $\sigma_{2} / \sigma_{1}=2$. Results are shown for three different well depth ratios ranging from $\epsilon_{2} / \epsilon_{1}=0.5$ to $\epsilon_{2} / \epsilon_{1}=1.5$.

low temperatures, although, for reasons cited earlier, the simulation data at these low temperature may also be inaccurate.

\section{CONCLUSIONS}

In this work we have developed equations of state for square-well dimer fluids, square-well dimer mixtures, square-well dimer/monomer mixtures, and square-well heteronuclear dumbbell fluids based on perturbation theory and

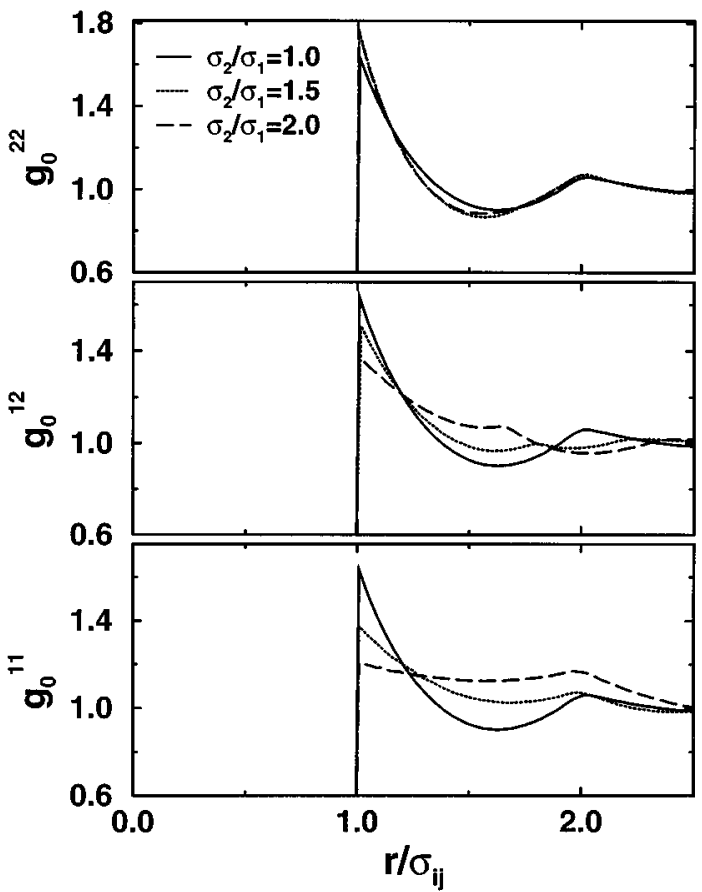

FIG. 13. The radial distribution functions $g_{0}^{11}, g_{0}^{12}$, and $g_{0}^{22}$ for equimolar hard-dimer mixtures at $\eta=0.3$ obtained from molecular dynamics simulation. The solid lines represent the radial distribution function for the pure dimer fluid $\left(\sigma_{2} / \sigma_{1}=1\right)$, the dotted and the dashed lines are the radial distribution functions for dimer mixtures at $\sigma_{2} / \sigma_{1}=1.5$ and 2 , respectively.

then tested them against discontinuous canonical molecular dynamics (DCMD) simulations. We have also conducted DCMD simulations of square-well monomer fluids and square-well monomer mixtures. The canonical molecular dynamics simulation method used here is a discontinuouspotential adaptation of Anderson's method. The DCMD method yields accurate compressibility factors for squarewell homonuclear monomer and dimer fluids which are in excellent agreement with previous MC simulations.

TABLE VI. $I_{0}^{i j}$ for hard dimer mixtures, hard dimer/monomer mixtures and hard heteronuclear dumbbells calculated from molecular dynamics simulations at $\eta=0.3$.

\begin{tabular}{|c|c|c|c|c|c|}
\hline \multirow[b]{2}{*}{ Dimer-dimer mix } & \multirow[b]{2}{*}{$I_{0}^{i j}$} & \multicolumn{2}{|c|}{$\sigma_{2} / \sigma_{1}=1.0$} & \multirow{2}{*}{$\frac{\sigma_{2} / \sigma_{1}=1.5}{\text { simulation }}$} & \multirow{2}{*}{$\frac{\sigma_{2} / \sigma_{1}=2.0}{\text { simulation }}$} \\
\hline & & fit & simulation & & \\
\hline & $I_{0}^{11}$ & 0.8842432 & 0.884189 & 0.875032 & 0.876668 \\
\hline & $I_{0}^{12}$ & 0.8842432 & 0.884189 & 0.896821 & 0.898199 \\
\hline & $I_{0}^{22}$ & 0.8842432 & 0.884189 & 0.886194 & 0.888621 \\
\hline \multirow[t]{4}{*}{ Dimer-monomer mix } & & \multicolumn{2}{|c|}{$\sigma_{d} / \sigma_{m}=1.0$} & $\sigma_{d} / \sigma_{m}=1.5$ & $\sigma_{d} / \sigma_{m}=2.0$ \\
\hline & $I_{0}^{d d}$ & 0.857521 & 0.8587109 & 0.859818 & 0.859447 \\
\hline & $I_{0}^{\text {md }}$ & 1.000742 & 0.999479 & 0.988196 & 0.969592 \\
\hline & $I_{0}^{m m}$ & 1.120648 & 1.121796 & 1.077587 & 1.058822 \\
\hline \multirow[t]{4}{*}{ Heteronuclear dumbbell } & & \multicolumn{2}{|c|}{$\sigma_{2} / \sigma_{1}=1.0$} & $\sigma_{2} / \sigma_{1}=1.5$ & $\sigma_{2} / \sigma_{1}=2.0$ \\
\hline & $I_{0}^{11}$ & 0.8842432 & 0.884189 & 0.792379 & 0.695192 \\
\hline & $I_{0}^{12}$ & 0.8842432 & 0.884189 & 0.876952 & 0.84740 \\
\hline & $I_{0}^{22}$ & 0.8842432 & 0.884189 & 0.957661 & 1.023424 \\
\hline
\end{tabular}


Our perturbation theory is based on Barker and Henderson's local compressibility approximation and Chang and Sandler's perturbation approach for monomers. The perturbation theory expressions for the compressibility factors of square-well dimers, square-well dimer-mixtures, square-well dimer-monomer mixtures and square-well heteronuclear dumbbells are derived. For mixtures we have used a conformal fluid mixing rule approach proposed by Henderson ${ }^{25}$ and Hino and Prausnitz ${ }^{26}$ to obtain average mixture properties.

In order to assess the accuracy of the new perturbation theory, the compressibility factor predictions of perturbation theory are compared to the compressibility factors obtained from DCMD simulation. The perturbation theory is found to accurately predict the compressibility factors of square-well dimer mixtures, dimer-monomer mixtures and square-well heteronuclear dumbbell fluids at intermediate densities for all cases including cases where both the relative well depth and the relative hard-core size of the two components are different. However, perturbation theory is found to overestimate the compressibility factor at high densities for mixtures where the hard-core diameters of the two components are considerably different. The perturbation theory is less accurate at low temperatures and at low densities. In this region the simulation data itself may also not be accurate because these states could be in the two-phase region.

\section{ACKNOWLEDGMENTS}

We would like to thank Dr. Yaoqi Zhou for helping us with the DCMD simulation algorithm. We would also like to thank Toshiaki Hino for providing his paper prior to publication and for very useful discussions. This work was supported by the Director, Office of Energy Research, Office of Basic Sciences, Chemical Sciences Division of the U.S. Department of Energy under contract number DE-FG0591ER1481. Acknowledgment is made to the Donors of the Petroleum Research Fund, administered by the American Chemical Society, for partial support of this research.

\section{APPENDIX: RADIAL DISTRIBUTION FUNCTIONS FOR HARD-DIMER MIXTURES}

Here we present the radial distribution functions obtained using molecular dynamics simulation of hard-dimer mixtures. Our motive is to check the accuracy of the approximations made in Eq. 34 that

$$
I_{0}^{i j} \equiv \int_{1}^{\lambda} g_{0}^{i j}(x) x^{2} \mathrm{~d} x \approx I_{0}^{\mathrm{dim}} \equiv \int_{1}^{\lambda} g_{0}^{\operatorname{dim}}(x) x^{2} \mathrm{~d} x,
$$

where $g_{0}^{i j}$ are the segment-segment radial distribution functions of hard-dimer mixtures, $x=r / \sigma_{i j}$ is the reduced distance, and $I_{0}^{\mathrm{dim}}$ is the integral of the radial distribution function of a homonuclear hard-dimer fluid $\left(g_{0}^{\mathrm{dim}}\right)$ at the same packing fraction as the mixture. Our hypothesis is that since the $g_{0}^{i j}$ are integrated over reduced units, the integrals $I_{0}^{i j}$ should be relatively independent of $\sigma_{i j}$. In order to check the accuracy of this approximation, we conducted molecular dynamics simulation to obtain the radial distribution functions of hard-dimer mixtures and hard dimer fluids. Figure
13 shows the $g_{0}^{i j}\left(g_{0}^{11}, g_{0}^{12}\right.$, and $\left.g_{0}^{22}\right)$ as a function of reduced distance $r / \sigma_{i j}$ for equimolar mixtures of hard-dimers at $\eta$ $=0.3$ for $\sigma_{2} / \sigma_{1}=1.5$ and 2 . The radial distribution function for the hard dimer fluid $\left(\sigma_{2} / \sigma_{1}=1\right)$ at $\eta=0.3$ is also shown. Although the shapes of these curves do depend on the value of $\sigma_{i j}$, the values of $I_{0}^{i j}$ obtained by numerical integration, shown in Table VI, are fairly insensitive to $\sigma_{i j}$. We find that, within simulation error, $I_{0}^{i j}$ for dimer mixtures with segments of different sizes can safely be approximated by $I_{0}^{i j}$ for the pure dimer fluid $\left(I_{0}^{\mathrm{dim}}\right)$.

Similar arguments can be used to calculate the integrals $I_{0}^{i j}$ for a hard dimer/monomer mixture and for a hard heteronuclear dumbbell fluid where the hard-core diameters of segments of the two components are different. The $I_{0}^{i j}$ for a hard dimer/monomer mixture with segments of different sizes can be approximated by the corresponding $I_{0}^{i j}$ for a hard dimer/ monomer mixture with segments of the same size. Similarly, the $I_{0}^{i j}$ for a hard-heteronuclear dumbbell fluid can be approximated by $I_{0}^{\mathrm{dim}}$, the integral of the radial distribution function over reduced units for a pure dimer fluid at the same packing fraction. Table VI shows the $I_{0}^{i j}$ for hard dimer/ monomer mixtures obtained via molecular dynamics simulation at $\eta=0.3$ for cases where $\sigma_{d} / \sigma_{m}=1,1.5$, and $2\left(x_{d}\right.$ $=0.333$ for all cases). Table VI also shows the $I_{0}^{i j}$ for hard heteronuclear dumbbell fluids obtained via molecular dynamics simulation at $\eta=0.3$ for the cases where $\sigma_{2} / \sigma_{1}=1,1.5$, and 2 . We observe that the values of $I_{0}^{i j}$ are relatively independent of the ratio of the hard core diameters of the two components except for heteronuclear dumbbell fluids.

${ }^{1}$ H. S. Gulati, J. M. Wichert, and C. K. Hall, J. Chem. Phys. 104, 5220 (1996)

${ }^{2}$ J. M. Wichert, H. S. Gulati, and C. K. Hall, J. Chem. Phys. 105, 7760 (1996).

${ }^{3}$ A. Rotenberg, J. Chem. Phys. 43, 1198 (1965).

${ }^{4}$ B. J. Alder, D. A. Young, and M. A. Mark, J. Chem. Phys. 56, 3013 (1972)

${ }^{5}$ Y. Rosenfeld and R. Thieberger, J. Chem. Phys. 63, 1875 (1975).

${ }^{6}$ D. Henderson, W. G. Madden, and D. D. Fitts, J. Chem. Phys. 64, 5026 (1976).

${ }^{7}$ J. A. Barker and D. Henderson, J. Chem. Phys. 47, 2856 (1967).

${ }^{8}$ W. R. Smith, D. Henderson, and Y. Tago, J. Chem. Phys. 47, 2856 (1977).

${ }^{9}$ K. D. Luks and J. J. Kozak, Advances in Chemical Physics (Wiley, New York, 1978).

${ }^{10}$ K. H. Lee, S. I. Sandler, and N. C. Patel, Fluid Phase Equilib. 25, 31 (1986).

${ }^{11}$ K.-H. Lee and S. I. Sandler, Fluid Phase Equilib. 34, 113 (1987).

${ }^{12}$ R. J. Lee and K. C. Chao, Mol. Phys. 61, 1431 (1987).

${ }^{13}$ M. Guo, W. Wang, and H. Lu, Fluid Phase Equilib. 60, 221 (1990).

${ }^{14}$ M. Guo, W. Wang, and H. Lu, Fluid Phase Equilib. 60, 37 (1990).

${ }^{15} \mathrm{H}$. Wang and W. Wang, Fluid Phase Equilib. 60, 11 (1990).

${ }^{16}$ C. P. Bokis and M. D. Donohue, Int. J. Thermophys. 16, 1277 (1995).

${ }^{17}$ A. Yethiraj and C. K. Hall, Mol. Phys. 72, 619 (1991).

${ }^{18}$ P. Vimalchand, A. Thomas, I. G. Economou, and M. D. Donohue, Fluid Phase Equilib. 73, 39 (1992).

${ }^{19}$ A. Thomas and M. D. Donohue, Mol. Phys. 81, 43 (1994).

${ }^{20}$ A. Yethiraj and C. K. Hall, J. Chem. Phys. 95, 8494 (1991).

${ }^{21}$ F. W. Tavares, J. Chang, and S. I. Sandler, Mol. Phys. 86, 1451 (1995).

${ }^{22}$ J. Chang and S. I. Sandler, Mol. Phys. 81, 745 (1994).

${ }^{23}$ T. Boublik, Mol. Phys. 27, 1415 (1974).

${ }^{24}$ T. Boublik, J. Chem. Phys. 63, 4084 (1975).

${ }^{25}$ D. Henderson, J. Chem. Phys. 61, 926 (1974).

${ }^{26}$ T. Hino and J. M. Prausnitz, Fluid Phase Equilib. (in press). 
${ }^{27}$ A. E. Sherwood and J. M. Prausnitz, J. Chem. Phys. 41, 429 (1964).

${ }^{28}$ D. Henderson, J. A. Barker, and W. R. Smith, J. Chem. Phys. 64, 4244 (1976).

${ }^{29}$ B. J. Alder and T. E. Wainwright, J. Chem. Phys. 31, 459 (1959).

${ }^{30}$ D. C. Rapaport, J. Phys. A 11, L213 (1978).

${ }^{31}$ D. C. Rapaport, J. Chem. Phys. 71, 3299 (1979).

${ }^{32}$ A. Bellemans, J. Orban, and D. V. Belle, Mol. Phys. 39, 781 (1980).

${ }^{33}$ H. C. Anderson, J. Chem. Phys. 72, 2384 (1980).

${ }^{34}$ Y. Zhou, C. K. Hall, and M. Karplus, Phys. Rev. Lett. 77, 2822 (1996).

${ }^{35}$ Y. Zhou, M. Karplus, J. M. Wichert, and C. K. Hall (in preparation).

${ }^{36}$ M. P. Allen and D. J. Tildesley, Computer Simulation of Liquids (Clarendon, Oxford, 1987).

${ }^{37}$ R. D. Present, Kinetic Theory of Gases (McGraw-Hill, New York, 1958).

${ }^{38}$ R. H. Fowler, Statistical Thermodynamics (Cambridge University Press, Cambridge, 1949).

${ }^{39}$ L. Verlet, Phys. Rev. 159, 98 (1967).

${ }^{40}$ S. W. Smith, B. D. Freeman, and C. K. Hall, J. Comput. Phys 134, 16 (1997).
${ }^{41}$ J. J. Erpenbeck and W. W. Wood, in Modern Theoretical Chemistry, edited by B. J. Berne (Plenum, New York, 1977).

${ }^{42}$ B. Quentrec and C. Brot, J. Comp. Phys. 13, 430 (1975).

${ }^{43}$ W. R. Smith, D. Henderson, and J. A. Barker, J. Chem. Phys. 53, 508 (1970).

${ }^{44}$ J. Chang and S. I. Sandler, Chem. Eng. Sci. 49, 2777 (1994).

${ }^{45}$ N. F. Carnahan and K. E. Starling, J. Chem. Phys. 51, 635 (1969).

${ }^{46}$ L. Vega, E. D. Miguel, L. F. Rull, G. Jackson, and L. A. McLure, J. Chem. Phys. 96, 296 (1992).

${ }^{47}$ D. J. Tildesley and W. B. Street, Mol. Phys. 41, 85 (1980).

${ }^{48}$ M. Chen, D. Henderson, and J. A. Barker, Can. J. Phys. 47, 2009 (1969).

${ }^{49}$ C. P. Bokis, M. D. Donohue, and C. K. Hall, J. Phys. Chem. 96, 11004 (1992).

${ }^{50}$ R. J. Lee and K. C. Chao, Mol. Phys. 65, 1253 (1988).

${ }^{51}$ J. M. Wichert and C. K. Hall (unpublished data, 1996). 